QUARTERLY OF APPLIED MATHEMATICS

VOLUME LXIV, NUMBER 4

DECEMBER 2006, PAGES 711-734

S 0033-569X(06)01042-3

Article electronically published on October 31, 2006

\title{
FREE VIBRATIONS OF A POLAR BODY AT ELASTIC RANGE
}

\author{
BY \\ GÜLAY ALTAY (Faculty of Engineering, Boḡaziçi University, Bebek, 34342 Istanbul, Turkey) \\ AND \\ M. CENGIZ DÖKMECI (Istanbul Technical University, P.K. 9, Gümüsuyu, 34430 Istanbul, Turkey)
}

\begin{abstract}
The purpose of this paper is to study certain features of the equations governing the time-harmonic free vibrations of a polar body at elastic range. The governing equations of micropolar elasticity are expressed in differential form, and then, the uniqueness of their solutions is investigated. The conditions sufficient for uniqueness are enumerated using the logarithmic convexity argument without any positive-definiteness assumptions of material elasticity. Applying a general principle of physics and modifying it through an involutory transformation, a unified variational principle is obtained that leads to all the governing equations of the free vibrations as its Euler-Lagrange equations. The governing equations are alternatively expressed in terms of the operators related to the kinetic and potential energies of the body. The basic properties of vibrations are studied and a variational principle in Rayleigh's quotient is given. As an application, the high-frequency vibrations of an elastic plate are treated.
\end{abstract}

1. Introduction. Polar theories of continua are introduced to predict the physical response of a class of materials possessing granular and fibrous structures or large molecules such as polymers. They constitute some extensions of the classical (or nonpolar) theory of elasticity that becomes inadequate in certain instances of polar materials (e.g., high-frequency short wavelength vibrations and ultrasonic waves). In the classical theory, the transmission of interaction between any two elements of material is assumed to be through the action of a force vector only (i.e., Cauchy's second law of motion). The transmission of interaction is postulated to be through the action of a force vector as well as a couple or moment vector, thus giving rise to the so-called polar (oriented or couple stress) theory of continua. In a polar theory, a material element of continua

Received February 17, 2006.

2000 Mathematics Subject Classification. Primary 74H25, 49R50, 74H45.

The authors acknowledge the financial support in part by their departments and TUBA, and the second author (M.C.D.) is grateful to Prof. Dr. Oral Büyüköztürk (Department of Civil and Environmental Engineering, Massachusetts Institute of Technology, Cambridge) for his kind invitation and support to the three-day workshop on engineering materials on June 9-11, 2004, Cambridge, Mass.

E-mail address: askarg@boun.edu.tr

E-mail address: cengiz.dokmeci@itu.edu.tr

(C)2006 Brown University 
that can support the couple stresses and the body couples has six degrees of freedom, a displacement vector and an independent microrotation vector, whereas it has three degrees of freedom in the nonpolar theory. A rational polar theory was first developed by the Cosserat brothers [1], and much later, in the nineteen sixties, many polar theories were proposed by some eminent scientists (e.g., [2] and the references therein). However, only a few experimental studies of a corroborative nature were conducted so as to determine the constitutive coefficients of polar materials (e.g., [3, 4]). This was largely due to the difficulty of isolating the effects of the couple stress and the spin inertia. A comprehensive review of polar theories was reported, including the historical background and developments to date and some applications (e.g., [5]-9]).

In this paper, the theory of micropolar continua is taken as a basis due to its deterministic, experimentally corroborated and generally accepted nature among the polar theories of continua at the elastic range [7. Most recently, the micropolar theory of elasticity was revisited, the physical significance of material elasticities was explained and some applications of the theory to composites and porous media were reviewed [10. In the theory, any material element is assumed to be endowed with an internal microstructure, and hence, the usual displacement vector and also, the microrotation vector characterizes the deformation of the material element. The microrotation vector, which is kinematically independent from the displacement vector, is introduced to describe the rotation of the microstructure within the material element. The governing equations of micropolar elasticity were stated in differential form by the divergence equations (i.e., first and second laws of motion), the gradient equations, the constitutive relations and the boundary and initial conditions. The internal consistency of the governing equations, that is, the existence and uniqueness of their solutions, was established for static and dynamic micropolar elastic solids, respectively (e.g., 7], [1], 12] and the references therein). Alternatively, the governing equations were expressed in variational form by some variational principles with their well-known features (e.g., 6], [7] and [13]-16]). The governing equations were used in both differential and variational forms in treating waves and vibrations in micropolar elements. An up-to-date critical account of micropolar elasticity was reviewed, including some 1-D and 2-D equations, variational principles and static and dynamic applications of elements [12, 17].

In view of the aforementioned reviews, this paper concerns the equations governing the time-harmonic free vibrations of a micropolar elastic body, including the uniqueness of solutions, some variational formulations and the fundamental properties of vibrations. The paper is organized as follows. In the next section, the governing equations of micropolar elasticity are summarized in differential form. In Section 3, the uniqueness is investigated in solutions of the governing equations, and the boundary and initial conditions sufficient to the uniqueness are enumerated by the logarithmic convexity argument. In Section 4, the principle of virtual work is applied to the time-harmonic free vibrations of a micropolar elastic body and a two-field variational principle is obtained. Then, modifying this principle by an involutory (or Legendre's) transformation, a unified variational principle is derived that has all the governing equations of vibrations as its Euler-Lagrange equations. In Section 5, an abstract formulation of the governing equations is presented by use of the operators related to the kinetic and potential energies 
of the body, and then, the basic properties of operators and vibrations are studied and Rayleigh's quotient is given. Section 6 is devoted to the high-frequency free vibrations of a micropolar elastic plate. The last section is devoted to some concluding remarks.

Notation. In this paper, standard indicial notation is freely used in the Euclidean 3-D space $\Xi$. Accordingly, Einstein's summation convention is implied for all repeated Latin indices $(1,2,3)$ and Greek indices $(1,2)$, unless they are enclosed within parentheses. A superposed dot stands for time differentiation and a comma for partial differentiation with respect to an indicated space coordinate $x_{i}$. Subscripts (n) and (m) are used to indicate a quantity belonging to the nonpolar field and the micropolar field, respectively. Furthermore, $C_{\alpha \beta}$ refers to a class of functions with derivatives of order up to and including $(\alpha)$ and $(\beta)$ with respect to $x_{i}$ and time $t$, respectively.

2. Governing equations of micropolar elasticity. In the Euclidean $3 \mathrm{D}$-space $\Xi$, let $\Omega+\partial \Omega$ with its smooth boundary surface $\partial \Omega$ and closure $\bar{\Omega}(=\Omega \cup \partial \Omega)$ denote the finite and bounded region of a micropolar elastic body. The regular region is referred to a system of Cartesian rectangular coordinates $x_{i}$ of the space. The Cartesian product of the region $\Omega(t)$ at time $t$ and the time interval $T=\left[t_{0}, t_{1}\right)$ is denoted by $\bar{\Omega} \times T$, which represents the domain of definitions for the micropolar elastic fields of functions of $\left(x_{i}, t\right)$. The equations governing the motion of an anisotropic micropolar but nonlocal as well as nonrelativistic elastic body may be grouped as the divergence and gradient equations, the constitutive relations, and the boundary and initial conditions to supplement them. Now, the governing equations are given (e.g., 7]), for ease of reference, in differential form by

Divergence equations. (Cauchy's first and second laws of motion)

$$
\begin{gathered}
t_{i j, i}+\rho\left(f_{j}-a_{j}\right)=0 \quad \text { in } \bar{\Omega} \times T, \\
m_{i j, i}+\varepsilon_{j k l} t_{k l}+\rho\left(l_{j}-b_{j}\right)=0 \quad \text { in } \bar{\Omega} \times T,
\end{gathered}
$$

where $t_{i j} \in C_{10}$ is the asymmetric stress tensor, $\rho \in C_{00}$ the mass density, $f_{i} \in C_{00}$ the body force vector, $a_{i}\left(=\ddot{u}_{i}\right) \in C_{12}$ the acceleration vector, $u_{i} \in C_{12}$ the displacement vector, $m_{i j} \in C_{10}$ the couple stress tensor, $l_{i} \in C_{00}$ the body couple vector, $b_{i}\left(=J_{i j} \ddot{\phi}_{j}\right) \in$ $C_{12}$ the microacceleration vector, $J_{i j}\left(=J_{j i}\right) \in C_{00}$ the microinertia tensor, $\phi_{i} \in C_{12}$ the microrotation vector, and $\varepsilon_{i j k}$ the alternating tensor.

Gradient equations.

$$
\begin{gathered}
e_{i j}=\left(u_{j, i}+\varepsilon_{j i k} \phi_{k}\right)=0 \quad \text { in } \bar{\Omega} \times T, \\
\varepsilon_{i j}=\phi_{i, j}=0 \quad \text { in } \bar{\Omega} \times T,
\end{gathered}
$$

where $e_{i j} \in C_{00}$ and $\varepsilon_{i j} \in C_{00}$ are the strain and microstrain tensors.

Constitutive relations.

$$
\begin{gathered}
t_{i j}=\frac{\partial u}{\partial e_{i j}} \quad \text { in } \bar{\Omega} \times T, \\
m^{i j}=\frac{\partial u}{\partial \varepsilon_{j i}} \quad \text { in } \bar{\Omega} \times T,
\end{gathered}
$$


where $u$ denotes the elastic energy density. Its quadratic form is expressed by

$$
u=\frac{1}{2}\left(A_{i j k l} e_{i j} e_{k l}+B_{i j k l} \varepsilon_{i j} \varepsilon_{k l}+2 C_{i j k l} e_{i j} \varepsilon_{k l}\right) \geq 0 .
$$

This yields the linear constitutive relations of the form

$$
\begin{gathered}
t_{i j}=A_{i j k l} e_{k l}+C_{i j k l} \varepsilon_{k l} \quad \text { in } \bar{\Omega} \times T, \\
m_{i j}=C_{k l j i} e_{k l}+B_{j i k l} \varepsilon_{k l} \quad \text { in } \bar{\Omega} \times T,
\end{gathered}
$$

where $A_{i j k l}=A_{k l i j}$ and $B_{i j k l}=B_{k l i j}$ refer to the isothermal elastic stiffnesses. If the micropolar body is isotropic, the material coefficients are specialized to

$$
\begin{aligned}
& A_{i j k l}=\lambda \delta_{i j} \delta_{k l}+(\mu+\kappa) \delta_{i k} \delta_{j l}+\mu \delta_{i l} \delta_{j k}, \\
& B_{i j k l}=\alpha \delta_{i j} \delta_{k l}+\beta \delta_{i l} \delta_{j k}+\gamma \delta_{i k} \delta_{j l}, \\
& C_{i j k l}=0
\end{aligned}
$$

in terms of Lamé's elasticity constants, $\lambda$ and $\mu$, and $\alpha, \beta, \gamma$ and $\kappa$, which are the four additional elastic moduli. Inserting (2.10) into (2.8) and (2.9), the linear constitutive relations are expressed by

$$
\begin{array}{cc}
t_{i j}=\lambda e_{k k} \delta_{i j}+(\mu+\kappa) e_{i j}+\mu e_{j i} & \text { in } \bar{\Omega} \times T, \\
m_{i j}=\alpha \varepsilon_{k k} \delta_{i j}+\beta \varepsilon_{i j}+\gamma \varepsilon_{j i}=0 & \text { in } \bar{\Omega} \times T,
\end{array}
$$

and also, the microinertia tensor becomes $J_{i j}=J \delta_{i j}$. The material coefficients satisfy the positive-semi-definite conditions of the form

$$
\begin{gathered}
3 \lambda+2 \mu+\kappa \geq 0,2 \mu+\kappa \geq 0, \kappa \geq 0,3 \alpha+\beta+\gamma \geq 0, \gamma+\beta \geq 0, \gamma-\beta \geq 0 \text { in } \bar{\Omega} \times T, \\
A_{i j k l} \eta_{i j} \eta_{k l} \geq 0, B_{i j k l} \eta_{i j} \eta_{k l} \geq 0
\end{gathered}
$$

for all nonzero tensors $\eta_{i j}$, and also, the microinertia tensor is positive-definite and the mass density is strictly positive, namely,

$$
\rho>0, \quad J_{i j} \eta_{i} \eta_{j} \geq 0
$$

for all nonzero vectors $\eta_{j}$.

Boundary conditions.

$$
\begin{gathered}
t_{j}^{*}=n_{i} t_{i j} \text { on } \partial \Omega_{t} \times T, \\
u_{i}=u_{i}^{*} \text { on } \partial \Omega_{u} \times T
\end{gathered}
$$

and

$$
\begin{gathered}
m_{j}^{*}=n_{i} m_{i j} \text { and } \partial \Omega_{m} \times T, \\
\phi_{i}=\phi_{i}^{*} \text { on } \partial \Omega_{\phi} \times T .
\end{gathered}
$$

Here, an asterisk is used to denote the prescribed quantities, $t_{j}=n_{i} t_{i j}$ and $m_{j}=n_{i} m_{i j}$ are the stress and couple stress vectors, respectively, $n_{i}$ is the unit outward vector normal to the boundary surface $\partial \Omega$, and $\left(\partial \Omega_{u}, \partial \Omega_{t}\right)$ and $\left(\partial \Omega_{\phi}, \partial \Omega_{m}\right)$ are its complementary regular sub-surfaces (i.e., $\partial \Omega_{u} \cup \partial \Omega_{t}=\partial \Omega$ and $\partial \Omega_{u} \cap \partial \Omega_{t}=\emptyset$ ). 
Initial conditions.

$$
\begin{array}{ll}
u_{i}\left(x_{j}, t_{0}\right)=v_{i}^{*}\left(x_{j}\right), w_{i}^{*}\left(x_{j}\right)=\dot{u}_{i}\left(x_{j}, t_{0}\right) & \text { in } \Omega\left(t_{0}\right), \\
\phi_{i}\left(x_{j}, t_{0}\right)=\varphi_{i}^{*}\left(x_{j}\right), \Phi_{i}^{*}\left(x_{j}\right)=\dot{\phi}_{i}\left(x_{j}, t_{0}\right) & \text { in } \Omega\left(t_{0}\right) .
\end{array}
$$

Governing equations. The foregoing 3-D equations completely describe the physical response of an anisotropic micropolar elastic continuum where the relativistic and quantum effects are excluded. The governing equations are deterministic, that is, there exist 42 equations for a state of solutions, $\wedge\left\{t^{i j} \in C_{10}, e_{i j} \in C_{00}, u_{i} \in C_{12} ; m^{i j} \in C_{10}, \varepsilon_{i j} \in\right.$ $\left.C_{00}, \phi_{i} \in C_{12}\right\}$, which contains 42 dependent variables of the functions of the space coordinates $x^{i}$ and time $t$. The boundary and initial conditions (2.16)-(2.21) are shown to be sufficient for a unique solution of the governing equations in the next section.

3. Uniqueness of solutions. The existence and uniqueness of solutions are of major concern for the governing equations of a continuum from both the physical and computational points of view. The first results on the uniqueness of solutions were credited to Kirchhoff and Neumannn in elastostatics and elastodynamics, respectively (e.g., [18]). Both the authors and many followers appealed to the energy argument in investigating the uniqueness in solutions of the governing equations of different materials under the mechanical, electrical and similar effects [19. Apart form the energy argument, other methods with their own merits are available in establishing the conditions for the uniqueness of solutions [20]. Of the methods, the logarithmic convexity argument was recently used so as to determine the conditions of uniqueness in poroelasticity 21]. The logarithmic convexity argument, contrary to the energy argument, does not impose any definiteness assumptions on the material elasticity. Now, this method with a mathematical basis is used in obtaining the conditions for a unique solution in micropolar elasticity.

In establishing the conditions of uniqueness, one considers, as usual, two possible states of solutions $\Lambda^{(\alpha)}$ and the difference state $\Lambda$ of solutions of the form

$$
\Lambda=\Lambda^{(2)}-\Lambda^{(1)}=\left\{u_{i}\left(=u_{i}^{(2)}-u_{i}^{(1)}\right), e_{i j}\left(=e_{i j}^{(2)}-e_{i j}^{(1)}\right), \ldots, m_{i j}\left(=m_{i j}^{(2)}-m_{i j}^{(1)}\right)\right\}
$$

for the 3-D governing equations of micropolar elastodynamics. The admissible states of solutions together with derivatives are assumed to exist, be single-valued and be continuously differentiable functions of the space coordinates $x_{i}$ and time $t$ in $\bar{\Omega} \times T$. The difference state of solutions evidently satisfies the homogeneous governing equations corresponding to zero body forces, body couples and prescribed surface tractions and deformations, by virtue of their linearity. Thus, one obtains the divergence equations (2.1) and (2.2) in the form

$$
\begin{gathered}
t_{i j, i}=\rho a_{j} \text { in } \bar{\Omega} \times T, \\
m_{i j, i}+\varepsilon_{j k l} t_{k l}=\rho b_{j} \text { in } \bar{\Omega} \times T,
\end{gathered}
$$


the gradient equations (2.3) and (2.4), the constitutive relations (2.8) and (2.9), the boundary conditions (2.16)-(2.19) given by

$$
\begin{gathered}
n_{i} t_{i j}=0 \text { on } \partial \Omega_{t} \times T, \quad u_{i}=0 \text { on } \partial \Omega_{u} \times T, \\
n_{i} m_{i j}=0 \text { on } \partial \Omega_{m} \times T, \quad \phi_{i}=0 \text { on } \partial \Omega_{\phi} \times T,
\end{gathered}
$$

and the initial conditions given by

$$
\begin{array}{cc}
u_{i}\left(x_{j}, t_{0}\right)=0, & \dot{u}_{i}\left(x_{j}, t_{0}\right)=0 \text { on } \Omega\left(t_{0}\right), \\
\phi_{i}\left(x_{j}, t_{0}\right)=0, & \dot{\phi}_{i}\left(x_{j}, t_{0}\right)=0 \text { in } \Omega\left(t_{0}\right) .
\end{array}
$$

Furthermore, the kinetic and potential energies, $K$ and $U$, for the micropolar region are given by

$$
K=\int_{\Omega} k d V, U=\int_{\Omega} u d V
$$

and in terms of the kinetic and potential energy densities, $k$ and $u$, are given by

$$
k=\frac{1}{2} \rho\left(\dot{u}_{i} \dot{u}_{i}+J_{i j} \dot{\phi}_{i} \dot{\phi}_{j}\right) \geq 0, \quad u=\frac{1}{2}\left(t_{i j} e_{i j}+m_{i j} \varepsilon_{i j}\right) \geq 0 .
$$

The total energy $\Sigma$ of the region is given by

$$
\Sigma=\int_{\Omega}(k+u) d V=K+U
$$

in terms of the difference state of solutions. The time rates of the kinetic and potential energies of the form

$$
\begin{gathered}
\dot{K}=\int_{\Omega} \rho\left(a_{i} \dot{u}_{i}+b_{i} \dot{\phi}_{i}\right) d V, \\
\dot{U}=\int_{\Omega}\left(t_{i j} \dot{e}_{i j}+m_{i j} \dot{\varepsilon}_{j i}\right) d V
\end{gathered}
$$

are readily obtained from (2.7), (3.8) and (3.9).

Now, multiplying the divergence equations (3.2) and (3.3) by $\dot{u}_{i}$ and $\dot{\phi}_{i}$, respectively, adding them and then integrating over the volume $\Omega$ of the micropolar region, one obtains

$$
\int_{\Omega}\left[t_{i j, i} \dot{u}_{j}+\left(m_{i j, i}+\varepsilon_{j k l} t_{k l}\right) \dot{\phi}_{j}\right] d V=\dot{K} .
$$

This may be written in the form

$$
\int_{\Omega}\left(t_{i j} \dot{u}_{j}+m_{i j} \dot{\phi}_{j}\right)_{, i} d V=\dot{K}+\int_{\Omega}\left(t_{i j} \dot{u}_{j, i}+m_{i j} \dot{\phi}_{j, i}+\varepsilon_{j l k} t_{k l} \dot{\phi}_{j}\right) d V .
$$

Remembering the divergence equations (2.3) and (2.4) and applying the divergence theorem to the regular micropolar region, (3.14) is expressed by

$$
B=\dot{K}+\dot{U}=\dot{\Sigma},
$$

where the boundary quantity of the form

$$
B=\int_{\Omega} n_{i}\left(t_{i j} \dot{u}_{j}+m_{i j} \dot{\phi}_{j}\right) d S
$$


is defined. By virtue of the boundary and initial conditions (3.4)-(3.7), and also, the specification of one member of each product, the integrand of the quantity and hence the quantity itself becomes zero. Thus, after integration with respect to time, (3.16) yields

$$
\Sigma\left(t_{1}\right)-\Sigma\left(t_{0}\right)=0 \text { in } \bar{\Omega} \times T \text {. }
$$

Because of the conservation of energy and the initial conditions (2.20) and (2.21), the integrand of (3.10) is nonnegative by definition and initially zero, provided that the condition (2.15) is met. Thus, the total energy of the micropolar region vanishes for the difference state of solutions for all time so that

$$
\Sigma(t)=0, \quad U=-K \text { in } \bar{\Omega} \times T
$$

is obtained.

The next step in the uniqueness of solutions requires the introduction of a logarithmic function of the form

$$
G(t)=\log F(t) ; F(t)=\frac{1}{2} \int_{\Omega} \rho\left(u_{i} u_{i}+J_{i j} \phi_{i} \phi_{j}\right) d V, t \in T
$$

in micropolar elasticity, as an extension of that in nonpolar elasticity [20]. The function $F(t)$ is evidently twice continuously differentiable in the time interval $T$, since the difference state of solutions satisfies the divergence equations (3.2) and (3.3). In addition, the integrand of the function $F(t)$, and hence the function itself, is nonnegative under the condition (2.15). However, the function may be equal to zero if its integrand is identically zero, that is, a zero deformation field $\left(u_{i}, \phi_{i}\right)$ being initially zero for $t \in T$. Thus, the uniqueness of solutions is readily implied by a trivial difference state of solutions $\Lambda$, that is, the condition $F(t)=0$ for all $t \in T$. Accordingly, without loss of generality, one may choose the function $F(t)$ as

$$
F(t)=0 \text { in } t_{0} \leq t \leq \tau_{1} \text { and } \tau_{2} \leq t \leq t_{1}, F(t)>0 \text { in } \tau_{1}<t<\tau_{2} \text { in } \bar{\Omega} \times T
$$

in the time interval $T=\left[t_{0}, t_{1}\right)$. It is shown that the function $G(t)$ is a convex function in the interval $\tau_{1}<t<\tau_{2}$ and the convexity is expressed by

$$
\ddot{G}=(\dot{F} / F)=\left(F \ddot{F}-\dot{F}^{2}\right) / F^{2} \geq 0
$$

or, equivalently,

$$
\Gamma_{c}=F^{2} \ddot{G}=F \ddot{F}-\dot{F}^{2} \geq 0 \text { in } \tau_{1}<t<\tau_{2}
$$

since $F(t)>0$.

To verify the convexity condition (3.20), the first and second derivatives of the function $F(t)$ are given in the form

$$
\begin{gathered}
\dot{F}(t)=\int_{\Omega} \rho\left(\dot{u}_{i} u_{i}+J_{i j} \dot{\phi}_{i} \phi_{j}\right) d V \\
\ddot{F}(t)=\int_{\Omega}\left[\rho\left(a_{i} u_{i}+b_{i} \phi_{i}\right)+\rho\left(\dot{u}_{i} \dot{u}_{i}+J_{i j} \dot{\phi}_{i} \dot{\phi}_{j}\right)\right] d V .
\end{gathered}
$$

Remembering the kinetic energy (3.8) and (3.9) and replacing the acceleration terms by those in (3.2) and (3.3), the second derivative may be expressed by

$$
\ddot{F}(t)=2 K+\int_{\Omega}\left[t_{i j, i} u_{j}+\left(m_{i j, i}+\varepsilon_{j k l} t_{k l}\right) \phi_{j}\right] d V
$$


and, equivalently, by

$$
\ddot{F}(t)=2 K+\int_{\Omega}\left[\left(t_{i j} u_{j}+m_{i j} \phi_{j}\right)_{, i}-\left(t_{i j} u_{j, i}+m_{i j} \phi_{j, i}-\varepsilon_{j k l} t_{k l} \phi_{j}\right)\right] d V .
$$

By applying the divergence theorem and using the gradient equations (2.3) and (2.4), (3.26) may also be expressed by

$$
\ddot{F}(t)=2 K-\int_{\Omega}\left(t_{i j} e_{i j}+m_{i j} \varepsilon_{j i}\right) d V+\int_{\partial \Omega} n_{i}\left(t_{i j} u_{j}+m_{i j} \phi_{j}\right) d S .
$$

In the right-hand side of this equation, the second term represents the potential energy given by (3.9) and (3.10), and the integrand of the last term vanishes under the boundary conditions (3.4) and (3.5). Thus, considering (3.18), the second derivative (3.27) of the form

$$
\ddot{F}(t)=2 K-2 U=4 K
$$

is finally written more concisely.

As a last step, substituting (3.19), (3.23) and (3.27) into the convexity condition in (3.28), we obtain

$$
\Gamma_{c}=\int_{\Omega} \mathbf{A D} d V \int_{\Omega} \dot{\mathbf{A}} \dot{\mathbf{D}} d V-\left\{\int_{\Omega}\{\dot{\mathbf{A}} \mathbf{D} d V\}^{2}\right.
$$

in terms of the matrices by

$$
\mathbf{A}=\left[\begin{array}{ll}
\rho u_{i} & \rho J_{i j} \phi_{i}
\end{array}\right], \quad \mathbf{D}=\left[\begin{array}{c}
u_{i} \\
\phi_{i}
\end{array}\right]
$$

with the identity of the form

$$
\mathbf{A} \dot{\mathbf{D}}=\dot{\mathbf{A} D} \text {. }
$$

By virtue of Schwartz's inequality, the convexity function is written as

$$
\Gamma_{c}=\frac{d^{2} G}{d t^{2}} \geq 0 \quad \text { in } \tau_{1}<t<\tau_{2}
$$

and hence, the convexity condition (3.22) is established. By integrating twice, the function $G(t)$ becomes

$$
G(t)=\log F(t)=C+C_{1} t \geq 0,
$$

where $C$ and $C_{1}$ are the integral constants. The constants are obtained from the continuity conditions (3.20) with the result

$$
F(t) \geq\left[F\left(\tau_{2}\right)\right]^{\alpha(t)}\left[F\left(\tau_{1}\right)\right]^{\beta(t)}, \quad \alpha(t)=\frac{t-\tau_{1}}{\tau_{2}-\tau_{1}}, \quad \beta(t)=\frac{\tau_{2}-t}{\tau_{2}-\tau_{1}} ; \quad \tau_{1} \leq t \leq \tau_{2} .
$$

Here, the condition $F\left(\tau_{1}\right)=0$ yields $F(t)=0$ for all $t \in\left[\tau_{1}, \tau_{2}\right]$, contrary to the hypothesis at the outset. Thus, $F(t)=0$ in the time interval $\tau_{1} \leq t \leq \tau_{2}$ and by continuity $F(t) \equiv 0$ in $T=\left[t_{0}, t_{1}\right)$. Remembering the existence and continuity assumptions of the micropolar field variables in $\bar{\Omega} \times T$, the difference state of solutions $\Lambda$ becomes trivial; that is, the states of solutions $\Lambda^{(1)}$ and $\Lambda^{(2)}$ are identical, and hence, a theorem of uniqueness is stated as follows. 
Theorem. In the Euclidean 3-D space $\Xi$, let $\Omega+\partial \Omega$ with its boundary surface $\partial \Omega$ and closure $\bar{\Omega}$ be a regular, finite and bounded micropolar elastic region with no singularities of any type. The region defines a motion that is maintained by the application of some assigned surface tractions and couples and, also, the prescribed deformation field over an appropriate portion of the piecewise smooth boundary surface $\partial \Omega$. Let an admissible state of solutions $\Lambda\left(u_{i} \in C_{12}, e_{i j} \in C_{00}, t_{i j} \in C_{10} ; \phi_{i} \in C_{12}, \varepsilon_{i j} \in C_{00}, m_{i j} \in C_{10}\right)$ exist and be continuously differentiable and single-valued functions of the space coordinates and time. Also, let the mass density be strictly positive and the microinertia tensor be positive-definite in $\bar{\Omega} \times T$. Then, the initial-mixed boundary value problem defined by the divergence equations (2.1) and (2.2), the gradient equations (2.3) and (2.4) and the linear constitutive relations (2.8) and (2.9) has at most one state of solutions $\Lambda$ if and only if this satisfies the boundary and initial conditions (2.16)-(2.21).

4. Variational principles for free vibrations. In Section 2, the governing equations of micropolar elasticity are expressed in differential form, and now, they are stated alternatively in variational form by certain variational principles. The variational principles with their well-known features are deduced from the principle of virtual work by modifying it through an involutory (or Legendre's) transformation. The principle of virtual work is, by definition, a differential type of variational principle (i.e., without an explicit functional) due to its postulated statements in terms of infinitesimals (i.e., virtual work, virtual displacements and the like). The principle and similar principles (e.g., the principle of virtual power, the principle of virtual dissipation) were used as a basis in deriving some unified variational principles of mechanics (e.g., 22]). An elaborate account of the principle of virtual work and related principles has been reported, including historical developments [23]. The principle of virtual work is stated for the vibrations of the micropolar elastic region as an assertion of the form

$$
\delta \tilde{L}=-\delta \tilde{U}-\delta \tilde{K}+\delta^{*} \tilde{W}=0
$$

with

$$
\delta(\tilde{U}, \tilde{K})=\delta \int_{\Omega}(\tilde{u}, \tilde{k}) d V, \quad \delta^{*} \tilde{W}=\int_{\partial \Omega}\left(\tilde{t}_{i}^{*} \delta \tilde{u}_{i}+\tilde{m}_{i}^{*} \delta \tilde{\phi}_{i}\right) d S .
$$

Here, $\tilde{L}$ is the Lagrangian function, $\delta^{*} \tilde{W}$ is the virtual work done by the external forces and it vanishes for the free vibrations, and an asterisk is placed upon $\delta^{*}$ in order to distinguish it from the variational operator $\delta$. A tilde is used to denote a quantity corresponding to the time-harmonic free vibrations with circular frequency $\omega$, namely,

$$
\begin{aligned}
& \left\{u_{i}\left(x^{j}, t\right), e_{i j}\left(x^{k}, t\right), \ldots, m_{i j}\left(x^{k}, t\right), \ldots\right\} \\
& \quad=\sum_{n=1}^{\infty} \exp i \omega_{n} t\left\{\tilde{u}_{i}\left(x^{j}\right), \tilde{e}_{i j}\left(x^{k}\right), \ldots, \tilde{m}_{i j}\left(x^{k}\right), \ldots\right\}^{(n)} .
\end{aligned}
$$

Henceforth, tildes are omitted for simplicity, the series expansion (4.2) is truncated at $N=1$ and only the first term with $\omega=\omega_{1}$ is retained.

Inserting (3.9) into (4.1) and recalling the fact that the variation operation may be commuted with the integration and differentiation operations in the region $\Omega+\partial \Omega$, and 
then executing the variations, using (2.5) and (2.6), and taking all the field quantities as given in (4.2), one obtains

$$
\delta L_{g}=-\int_{\Omega}\left(t_{i j} \delta e_{i j}+m_{i j} \delta \varepsilon_{j i}\right) d V+\int_{\Omega} \rho \lambda\left(u_{i} \delta u_{i}+J_{i j} \phi_{i} \delta \phi_{j}\right) d V=0, \lambda=\omega^{2} .
$$

In this equation, substituting the gradient equations (2.3) and (2.4), applying the divergence theorem and combining the terms on the surface and volume integrals, a two-field variational principle is obtained in the form

$$
\begin{aligned}
& \delta L_{g}\left\{\Lambda_{g}=u_{i}, \phi_{i}\right\}=\int_{\Omega}\left(t_{i j, i}+\rho \lambda u_{j}\right) \delta u_{j} d V+\int_{\Omega}\left(m_{i j, i}+\varepsilon_{j k l} t_{k l}+\rho \lambda J_{i j} \phi_{i}\right) \delta \phi_{j} d V \\
& -\int_{\partial \Omega} n_{j}\left(t_{j i} \delta u_{i}+m_{j i} \delta \phi_{i}\right) d S=0 .
\end{aligned}
$$

The variational principle (4.4) leads, as its Euler-Lagrange equations, to the divergence equations of the form

$$
\begin{gathered}
t_{i j, i}+\rho \lambda u_{j}=0 \quad \text { in } \bar{\Omega}, \\
m_{i j, i}+\varepsilon_{j k l} t_{k l}+\rho \lambda J_{j i} \phi_{i}=0 \quad \text { in } \bar{\Omega},
\end{gathered}
$$

and the associated natural boundary conditions (2.16) and (2.18) with $t_{i}^{*}$ and $m_{i}^{*}$ being zero for the free vibrations. The rest of the governing equations remain as the constraint (or subsidiary) conditions of the two-field variational principle (4.4). The constraint conditions prevent a free and simple choice of the coordinate (or trial, shape, approximating) functions and also, a simultaneous approximation upon all the field variables. Accordingly, the constraint conditions are now relaxed by an involutory (or Legendre's) transformation that is applicable to both holonomic and nonholonomic conditions (e.g., 24]). Of the methods of relaxation (e.g., [25]), Legendre's transformation is of wide use in elasticity and electroelasticity due to its versatility and clarity in application, and it is used herein as well.

To incorporate the remaining governing equations into the variational principle, the dislocation potentials of the form

$$
\begin{gathered}
\Delta_{1}^{1}=\int_{\Omega} \lambda_{i j}\left[e_{i j}-\left(u_{j, i}+\varepsilon_{j i k} \phi_{k}\right)\right] d S+\int_{\Omega} \mu_{i j}\left(\varepsilon_{i j}-\phi_{i, j}\right) d S, \\
\Delta_{2}^{2}=\int_{\partial \Omega_{u}} \lambda_{i} u_{i} d S+\int_{\partial \Omega_{\phi}} \mu_{i} \phi_{i} d S
\end{gathered}
$$

are introduced for the volume and surface constraint conditions, (2.3), (2.4), (2.17) and (2.19), and they are added into (4.1), namely,

$$
\delta L_{g}=\delta L+\delta \Delta_{\alpha}^{\alpha}=0 .
$$

Here, $\lambda^{i j}, \lambda^{i}, \mu^{i j}$ and $\mu^{i}$ are the Lagrangian multipliers to be determined, and they are regarded as independent variables. In a manner similar to that used in deriving the variational principle (4.4), executing the indicated variations, applying the divergence theorem to the regular region $\Omega+\partial \Omega$ and then combining the surface and volume terms, one arrives at the variational equation as

$$
\delta L_{g}=\delta L_{n}+\delta L_{p}=0
$$


with the denotations of the form

$$
\begin{aligned}
& \delta L_{n}=\int_{\Omega}\left\{\left(\lambda_{i j, i}+\rho \lambda u_{j}\right) \delta u_{j}+\left[e_{i j}-\left(u_{j, i}+\varepsilon_{j k l} \phi_{k}\right)\right] \delta \lambda_{i j}+\left(\lambda_{i j}-\frac{\partial u}{\partial e_{i j}}\right) \delta e_{i j}\right\} d V \\
& -\int_{\partial \Omega_{t}} n_{j} \lambda_{j i} \delta u_{i} d S+\int_{\partial \Omega_{u}}\left[u_{i} \delta \lambda_{i}+\left(\lambda_{i}-n_{j} \lambda_{j i}\right) \delta u_{i}\right] d S
\end{aligned}
$$

and

$$
\begin{aligned}
& \delta L_{p}=\int_{\Omega}\left\{\left(\mu_{i j, i}-\lambda_{k l} \varepsilon_{j k l}+\rho \lambda J_{i j} \phi_{i}\right) \delta \phi_{j}+\left(\varepsilon_{i j}-\phi_{i, j}\right) \delta \mu_{i j}+\left(\mu_{i j}-\frac{\partial u}{\partial \varepsilon_{i j}}\right) \delta \varepsilon_{i j}\right\} d V \\
& -\int_{\partial \Omega_{m}} n_{j} \mu_{j i} \delta \phi_{i} d S+\int_{\partial \Omega_{\phi}}\left[\phi_{i} \delta \mu_{i}+\left(\mu_{i}-n_{j} \mu_{i j}\right) \delta \phi_{i}\right] d S
\end{aligned}
$$

From (4.11) and (4.12), the Lagrangian multipliers are identified as

$$
\lambda_{i j}=\frac{\partial u}{\partial e_{i j}}=t_{i j}, \quad \lambda_{i}=n_{j} \lambda_{j i}=n_{j} t_{j i} ; \mu_{i j}=\frac{\partial u}{\partial \varepsilon_{i j}}=m_{i j}, \quad \mu_{i}=n_{j} \mu_{j i}=n_{j} m_{j i}
$$

by use of the fundamental lemma of the calculus of variations. Substituting the Lagrangian multipliers (4.13) into (4.10) and using (2.8) and (2.9), a six-field variational principle, namely,

$$
\delta L_{d}\left\{\Lambda_{d}=\Lambda_{n} \cup \Lambda_{p}\right\}=\delta L_{n}\left\{\Lambda_{n}=\left(u_{i}, e_{i j}, t_{i j}\right)\right\}+\delta L_{p}\left\{\Lambda_{p}=\left(\phi_{i}, \varepsilon_{i j}, m_{i j}\right)\right\}=0
$$

with

$$
\begin{aligned}
& \delta L_{n}\left\{\Lambda_{n}\right\}=\int_{\Omega}\left\{\left(t_{i j, i}+\rho \lambda u_{j}\right) \delta u_{j}+\left[e_{i j}-\left(u_{j, i}+\varepsilon_{j i k} \phi_{k}\right)\right] \delta t_{i j}\right. \\
& \left.+\left[t_{i j}-\left(A_{i j k l} e_{k l}+C_{i j k l} \varepsilon_{k l}\right)\right] \delta e_{i j}\right\}-\int_{\partial \Omega_{u}} u_{i} n_{j} \delta t_{j i} d S+\int_{\partial \Omega_{t}} n_{j} t_{j i} \delta u_{i} d S
\end{aligned}
$$

for the nonpolar part and

$$
\begin{aligned}
& \delta L_{m}\left\{\Lambda_{m}\right\}=\int_{\Omega}\left\{\left(m_{i j, i}+\varepsilon_{j k l} t_{k l}+\rho \lambda J_{i j} \phi_{i}\right) \delta \phi_{j}+\left(\varepsilon_{i j}-\phi_{j, i}\right) \delta m_{i j}\right. \\
& \left.+\left[m_{i j}-\left(C_{k l j i} e_{k l}+B_{j i k l} \varepsilon_{k l}\right)\right] \delta \varepsilon_{i j}\right\} d V+\int_{\partial \Omega_{m}} n_{j} m_{j i} \delta \phi_{i} d S-\int_{\partial \Omega_{\phi}} n_{j} \phi_{i} \delta m_{j i} d S
\end{aligned}
$$

for the micropolar part is obtained for the free vibrations of the elastic region $\Omega+\partial \Omega$. From (4.14), the three-field variational principles of the form

$$
\begin{gathered}
\delta L_{n}\left\{\Lambda_{n}=\left(u_{i}, e_{i j}, t_{i j}\right)\right\}=0, \\
\delta L_{m}\left\{\Lambda_{m}=\left(\phi_{i}, \varepsilon_{i j}, m_{i j}\right)\right\}=0
\end{gathered}
$$

are readily obtained for the nonpolar and micropolar parts, respectively. These variational principles are reciprocals of each other.

The six-field variational principle (4.14) yields as its Euler-Lagrange equations all the equations governing the free vibrations of the micropolar elastic region. They are the gradient equations (2.3) and (2.4), the linear constitutive relations (2.8) and (2.9), the divergence equations (4.5) and (4.6), and the boundary conditions

$$
u_{i}=0 \text { on } \partial \Omega_{u}, \quad n_{i} t_{i j}=0 \text { on } \partial \Omega_{t} ; \quad \phi_{i}=0 \text { on } \partial \Omega_{\phi}, \quad n_{i} m_{i j}=0 \text { on } \partial \Omega_{m} .
$$

The variational principle may be expressed by an explicit functional of the form

$$
\begin{aligned}
& L_{d}\left\{u_{i}, e_{i j}, t_{i j} ; \phi_{i}, \varepsilon_{i j}, m_{i j}\right\} \\
& =\int_{\Omega}\left\{-u-k+\left[e_{i j}-\left(u_{j, i}+\varepsilon_{j i k} \phi_{k}\right)\right] t_{i j}+\left(\varepsilon_{i j}-\phi_{i, j}\right) m_{i j}\right\} d V \\
& \quad+\int_{\partial \Omega_{u}} u_{i} n_{j} t_{i j} d S+\int_{\partial \Omega_{\phi}} \phi_{i} n_{j} m_{i j} d S .
\end{aligned}
$$


The validity of the variational principle (4.19) can be readily demonstrated; its EulerLagrange equations coincide with the governing equations of vibrations. The variational principle with its explicit functional represents an integral type of variational principles with all the well-known features of variational principles. It operates on all the micropolar field variables and yields, as special cases, the variational principles operating on some of the field variables. The variational principle is the counterpart of the Hellinger-Reissner variational principle in elasticity for the free vibrations in micropolar elasticity, and it is stated as follows.

Unified variational principle. Let the functional $L_{d}\left\{\Lambda_{d}\right\}$ in Eq. (4.19) be defined for the regular, finite and bounded micropolar elastic region $\Omega+\partial \Omega$ with its piecewise smooth boundary surface $\partial \Omega\left(=\partial \Omega_{t} \cup \partial \Omega_{u}=\partial \Omega_{m} \cup \partial \Omega_{\phi}\right.$ and $\left.\partial \Omega_{t} \cap \partial \Omega_{u}=\partial \Omega_{m} \cup \partial \Omega_{\phi}=\emptyset\right)$ and closure $\bar{\Omega}$ in the Euclidean 3-D space $\Xi$. Then, all the admissible states $\Lambda_{d}$ that satisfy the suitable existence, continuity and differentiability conditions of the micropolar field variables are only those that admit $\delta L_{d}\left\{\Lambda_{d}\right\}=0$ if and only if they satisfy the divergence equations (4.5) and (4.6), the gradient equations (2.3) and (2.4), the constitutive relations (2.5) and (2.6), and the boundary conditions (4.18) as the appropriate EulerLagrange equations. Conversely, if these governing equations of vibrations are identically met, the six-field variational principle (4.19) is evidently satisfied.

5. Free vibrations of a micropolar elastic body. Certain variational principles are derived for the time-harmonic free vibrations of the elastic micropolar region $\Omega+\partial \Omega$ in the previous section, and the governing equations are expressed by (4.14) in variational form, and also, by the divergence equations (4.4) and (4.5), the gradient equations (2.3) and (2.4), the linear constitutive relations (2.8) and (2.9) and the boundary conditions (4.18) in differential form. Now, inserting (2.3) and (2.4) into (2.8) and (2.9), and then, into (4.5) and (4.6) and (4.18), one obtains the divergence equations as

$$
\begin{gathered}
-A_{i j k l}\left(u_{l, k i}+\varepsilon_{l k s} \phi_{s, i}\right)-C_{i j k l} \phi_{k, l i}=\rho \lambda u_{j} \quad \text { in } \bar{\Omega}, \\
-C_{k l j i}\left(u_{l, k i}+\varepsilon_{l k s} \phi_{s, i}\right)-B_{j i k l} \phi_{k, l i}-\varepsilon_{j k l}\left[A_{k l r s}\left(u_{s, r}+\varepsilon_{s r i} \phi_{i}\right)+C_{k l r s} \phi_{r, s}\right] \\
=\rho \lambda J_{i j} \phi_{i} \quad \text { in } \bar{\Omega},
\end{gathered}
$$

and the boundary conditions as

$$
\begin{aligned}
& u_{i}=0 \text { on } \partial \Omega_{u},\left[A_{i j k l}\left(u_{l, k}+\varepsilon_{l k s} \phi_{s}\right)+C_{i j k l} \phi_{k, l}\right] n_{i}=0 \text { on } \partial \Omega_{t}, \\
& \phi_{i}=0 \text { on } \partial \Omega_{\phi},\left[C_{k l j i}\left(u_{l, k}+\varepsilon_{l k s} \phi_{s}\right)+B_{i j k l} \phi_{k, l}\right] n_{i}=0 \text { on } \partial \Omega_{m}
\end{aligned}
$$

in terms of the deformation components $\left(u_{i}, \phi_{i}\right)$. Equations (5.1) and (5.2) define an eigenvalue problem so as to determine the values of the parameter for which a nontrivial solution $u_{i}\left(x_{j}\right)$ and $\phi_{i}\left(x_{j}\right)$ exists for the region under the boundary conditions (5.3) and (5.4).

The eigenvalue problem, paralleling Yang and Batra [26] who examined the vibrations of a piezoelectric body, may be alternatively expressed in an abstract formulation by

$$
\mathbf{P}\{\mathbf{U}\}=\lambda \mathbf{K}\{\mathbf{U}\} \quad \text { in } \bar{\Omega} .
$$


That is, find $\lambda$ for which there exists a nontrivial solution under the boundary conditions

$$
u_{i}=0 \text { on } \partial \Omega_{u}, \quad \mathbf{T}\{\mathbf{U}\} n_{i}=0 \text { on } \partial \Omega_{t} ; \quad \phi_{i}=0 \text { on } \partial \Omega_{\phi}, \quad \mathbf{M}\{\mathbf{U}\} n_{i}=0 \text { on } \partial \Omega_{m} .
$$

Here, the operators $\mathbf{P}$ and $\mathbf{K}$, related to the potential and kinetic energies of the region by

$$
\begin{gathered}
\mathbf{P}\{\mathbf{U}\}=-\left\{A_{i j k l}\left(u_{l, k i}+\varepsilon_{l k s} \phi_{s, i}\right)+C_{i j k l} \phi_{k, l i} ;\right. \\
\left.C_{k l j i}\left(u_{l, k i}+\varepsilon_{l k s} \phi_{s, i}\right)+B_{j i k l} \phi_{k, l i}+\varepsilon_{j k l}\left[A_{k l r s}\left(u_{s, r}+\varepsilon_{s r i} \phi_{i}\right)+C_{k l r s} \phi_{r, s}\right]\right\}, \\
\mathbf{K}\{\mathbf{U}\}=\left\{\rho u_{i}, \rho J_{i j} \phi_{j}\right\}
\end{gathered}
$$

and the operators by

$$
\begin{aligned}
\mathbf{T}\{\mathbf{U}\} & =A_{i j k l}\left(u_{l, k}+\varepsilon_{l k s} \phi_{s}\right)+C_{i j k l} \phi_{k, l}, \\
\mathbf{M}\{\mathbf{U}\} & =C_{k l j i}\left(u_{l, k}+\varepsilon_{l k s} \phi_{s}\right)+B_{i j k l} \phi_{k, l},
\end{aligned}
$$

are defined. Also, the admissible function spaces that satisfy (5.1)-(5.4) as

$$
\mathbf{U}=\left\{u_{i}, \phi_{i}\right\}, \quad \Gamma\{\mathbf{U}\}=\mathbf{U} \cup \overline{\mathbf{U}}
$$

are introduced. In this equation, an overbar is used to indicate the complex conjugate of a quantity. The eigenvalue $\bar{\lambda}$ corresponds to $\overline{\mathbf{U}}$. Accordingly, (5.5) of the form

$$
\mathbf{P}\{\overline{\mathbf{U}}\}=\bar{\lambda} \mathbf{K}\{\overline{\mathbf{U}}\} \text { in } \bar{\Omega}
$$

is given.

Certain properties of the operators. Both the operators $\mathbf{P}\{\mathbf{U}\}$ and $\mathbf{K}\{\mathbf{U}\}$ are nonnegative and selfadjoint on the function space $\Gamma\{\mathbf{U}\}$. To show these properties, the kinetic and potential energies of the micropolar elastic region are computed from (2.7) and (3.9) as

$$
\begin{gathered}
U=\frac{1}{2} \kappa^{2} \int_{\Omega}\left(A_{i j k l} e_{i j} e_{k l}+B_{i j k l} \varepsilon_{i j} \varepsilon_{k l}+2 C_{i j k l} e_{i j} \varepsilon_{k l}\right) d V \geq 0, \\
K=\frac{1}{2} \kappa^{2} \int_{\Omega} \rho \lambda\left(u_{i} u_{i}+J_{i j} \phi_{i} \phi_{j}\right) d V \geq 0
\end{gathered}
$$

and, also, the condition is stated as

$$
K=\frac{1}{2} \int_{\Omega} \rho \lambda\left(u_{i} \bar{u}_{i}+J_{i j} \phi_{i} \bar{\phi}_{j}\right) d V \geq 0,
$$

where $\kappa$ is used to indicate a cosine mode or a sine mode (i.e., $\kappa=\cos \omega t$ or $\sin \omega t$ ). Now, one considers the inner product of the operator $\mathbf{P}\{\mathbf{U}\}$ and the function space $\mathbf{U}$, namely,

$$
\begin{aligned}
& \langle\mathbf{P}\{\mathbf{U}\}: \mathbf{U}\rangle=-\int_{\Omega}\left\{\left[A_{i j k l}\left(u_{l, k i}+\varepsilon_{l k s} \phi_{s, i}\right)+C_{i j k l} \phi_{k, l i}\right] u_{j}+\left[C_{i j k l}\left(u_{l, k i}+\varepsilon_{l k s} \phi_{s, i}\right)\right.\right. \\
& \left.\left.+B_{i j k l} \phi_{k, l i}\right] \phi_{j}+\varepsilon_{j k l}\left[A_{k l r s}\left(u_{s, r}+\varepsilon_{s r i} \phi_{i}\right)+C_{k l r s} \phi_{r, s}\right] \phi_{j}\right\} d V,
\end{aligned}
$$


which is readily expressed by

$$
\begin{aligned}
& \langle\mathbf{P}\{\mathbf{U}\}: \mathbf{U}\rangle=-\int_{\Omega}\left\{\left[A_{i j k l}\left(u_{l, k}+\varepsilon_{l k s} \phi_{s}\right)+C_{i j k l} \phi_{k, l}\right] u_{j}\right\}_{, i} d V \\
& +\int_{\Omega}\left[A_{i j k l}\left(u_{l, k}+\varepsilon_{l k s} \phi_{s}\right)+C_{i j k l} \phi_{k, l}\right] u_{j, i} d V \\
& -\int_{\Omega}\left\{\left[C_{i j k l}\left(u_{l, k}+\varepsilon_{l k s} \phi_{s}\right)+B_{i j k l} \phi_{k, l}\right] \phi_{j}\right\}_{, i} d V \\
& +\int_{\Omega}\left[C_{i j k l}\left(u_{l, k i}+\varepsilon_{l k s} \phi_{s, i}\right)+B_{i j k l} \phi_{k, l i}\right] \phi_{j, i} d V \\
& -\int_{\Omega} \varepsilon_{j k l}\left[A_{k l r s}\left(u_{s, r}+\varepsilon_{s r i} \phi_{i}\right)+C_{k l r s} \phi_{s, r}\right] \phi_{j} d V .
\end{aligned}
$$

Applying the divergence theorem to the regular region $\Omega+\partial \Omega$, (5.16) is written as

$$
\begin{aligned}
& \langle\mathbf{P}\{\mathbf{U}\}: \mathbf{U}\rangle=-\int_{\partial \Omega} \mathbf{T}\{\mathbf{U}\} n_{i} u_{j} d S-\int_{\partial \Omega} \mathbf{M}\{\mathbf{U}\} n_{i} \phi_{j} d S \\
& +\int_{\Omega}\left(A_{i j k l} e_{k l}+C_{i j k l} \varepsilon_{k l}\right) u_{j, i} d V \\
& +\int_{\Omega}\left(C_{k l j i} e_{l k}+B_{i j k l} \varepsilon_{k l}\right) \phi_{j, i} d V-\int_{\Omega} \varepsilon_{j k l}\left(A_{k l r s} e_{r s}+C_{k l r s} \varepsilon_{r s}\right) \phi_{j} d V,
\end{aligned}
$$

where the gradient equations (2.3) and (2.4) are used. In the right-hand side of (5.17), the first two terms vanish due to (5.3), (5.4) and (5.6) and the last three terms are combined as

$$
\langle\mathbf{P}\{\mathbf{U}\}: \mathbf{U}\rangle=\int_{\Omega}\left(A_{i j k l} e_{i j} e_{k l}+B_{i j k l} \varepsilon_{i j} \varepsilon_{k l}+2 C_{i j k l} e_{i j} \varepsilon_{k l}\right) d V .
$$

Likewise, the inner product of the operator $\mathbf{K}\{\mathbf{U}\}$ and $\mathbf{U}$ is expressed by

$$
\langle\mathbf{K}\{\mathbf{U}\}: \mathbf{U}\rangle=\int_{\Omega} \rho\left(u_{i} u_{i}+J_{i j} \phi_{i} \phi_{j}\right) d V .
$$

In view of (5.13) and (5.14), (5.18) and (5.19) are stated in the form

$$
\langle\mathbf{P}\{\mathbf{U}\}: \mathbf{U}\rangle \geq 0, \quad\langle\mathbf{K}\{\mathbf{U}\}: \mathbf{U}\rangle \geq 0,
$$

which shows that the operators $\mathbf{P}\{\mathbf{U}\}$ and $\mathbf{K}\{\mathbf{U}\}$ are nonnegative on the function space $\mathbf{U}\left\{u_{i}, \phi_{i}\right\}$.

As before, the inner product of the operator $\mathbf{P}\{\mathbf{U}\}$ and a function space $\mathbf{V}\left\{v_{i}, \varphi_{i}\right\} \in \Gamma$ is expressed by

$$
\begin{aligned}
& \langle\mathbf{P}\{\mathbf{U}\}: \mathbf{V}\rangle=-\int_{\Omega}\left\{\left[A_{i j k l}\left(u_{l, k i}+\varepsilon_{l k s} \phi_{s, i}\right)+C_{i j k l} \phi_{k, l i}\right] v_{j}\right. \\
& +\left[C_{i j k l}\left(u_{l, k i}+\varepsilon_{l k s} \phi_{s, i}\right)+B_{i j k l} \phi_{k, l i}\right] \varphi_{j} \\
& \left.+\varepsilon_{j k l}\left[A_{k l r s}\left(u_{s, r}+\varepsilon_{s r i} \phi_{i}\right)+C_{k l r s} \phi_{r, s}\right] \varphi_{j}\right\}_{, i} d V,
\end{aligned}
$$

which is readily written as

$$
\begin{aligned}
& \langle\mathbf{P}\{\mathbf{U}\}: \mathbf{V}\rangle=-\int_{\Omega}\left\{\left[A_{i j k l}\left(u_{l, k}+\varepsilon_{l k s} \phi_{s}\right)+C_{i j k l} \phi_{k, l}\right] v_{j}\right\}_{, i} d V \\
& +\int_{\Omega}\left[A_{i j k l}\left(u_{l, k}+\varepsilon_{l k s} \phi_{s}\right)+C_{i j k l} \phi_{k, l}\right] v_{j, i} d V \\
& -\int_{\Omega}\left\{\left[C_{i j k l}\left(u_{l, k}+\varepsilon_{l k s} \phi_{s}\right)+B_{i j k l} \phi_{k, l}\right] \varphi_{j}\right\}_{, i} d V \\
& +\int_{\Omega}\left[C_{i j k l}\left(u_{l, k}+\varepsilon_{l k s} \phi_{s, i}\right)+B_{i j k l} \phi_{k, l}\right] \varphi_{j, i} d V \\
& -\int_{\Omega} \varepsilon_{j k l}\left[A_{k l r s}\left(u_{s, r}+\varepsilon_{s r i} \phi_{i}\right)+C_{k l r s} \phi_{s, r}\right] \varphi_{j} d V .
\end{aligned}
$$

Applying the divergence theorem to the regular region and using (5.11) and (5.12), this equation becomes

$$
\langle\mathbf{P}\{\mathbf{U}\}: \mathbf{V}\rangle=-\int_{\partial \Omega} \mathbf{T}\{\mathbf{U}\} n_{i} v_{j} d S-\int_{\partial \Omega} \mathbf{M}\{\mathbf{U}\} n_{i} \varphi_{j} d S+\langle\mathbf{P}\{\mathbf{V}\}: \mathbf{U}\rangle .
$$

By virtue of the boundary conditions (5.3), (5.4) and (5.6), one finally obtains

$$
\langle\mathbf{P}\{\mathbf{U}\}: \mathbf{V}\rangle=\langle\mathbf{P}\{\mathbf{V}\}: \mathbf{U}\rangle .
$$


Similarly, the inner product of the operator $\mathbf{K}\{\mathbf{U}\}$ and the function space $\mathbf{V}\left\{v_{i}, \varphi_{i}\right\} \in \Gamma$ reads

$$
\langle\mathbf{K}\{\mathbf{U}\}: \mathbf{V}\rangle=\int_{\Omega} \rho\left(u_{i} v_{i}+J_{i j} \phi_{i} \varphi_{j}\right) d V
$$

which is written as

$$
\langle\mathbf{K}\{\mathbf{U}\}: \mathbf{V}\rangle=\langle\mathbf{K}\{\mathbf{V}\}: \mathbf{U}\rangle .
$$

Equations (5.24) and (5.26) indicate the selfadjointness of the operators $\mathbf{P}\{\mathbf{U}\}$ and $\mathbf{K}\{\mathbf{U}\}$ on the function space $\Gamma$.

In the case of a frequency shift due to some small changes in the physical and geometrical parameters, the eigenvalues and the eigenfunctions in (5.5) and the operators $\mathbf{P}\{\mathbf{U}\}$ and $\mathbf{K}\{\mathbf{U}\}$ may be expressed by the perturbation expansions in terms of a small parameter $\varepsilon$ as

$$
\lambda=\sum_{n=0}^{\infty} \varepsilon^{n} \lambda_{n}, \quad \mathbf{U}=\sum_{n=0}^{\infty} \varepsilon^{n} \mathbf{U}_{n} ; \quad \mathbf{P}=\sum_{n=0}^{\infty} \varepsilon^{n} \mathbf{P}_{n}, \quad \mathbf{K}=\sum_{n=0}^{\infty} \varepsilon^{n} \mathbf{K}_{n} ; \varepsilon<<1,
$$

where $\lambda_{n}$ and $\mathbf{U}_{n}$ are unknowns to be determined. Inserting the expansions (5.27) into (5.5), one obtains

$$
\mathbf{P}_{0}\left\{\mathbf{U}_{0}\right\}=\lambda_{0} \mathbf{K}_{0}\left\{\mathbf{U}_{0}\right\} \text { in } \bar{\Omega}
$$

and

$$
\mathbf{P}_{0}\left\{\mathbf{U}_{1}\right\}+\mathbf{P}_{0}\left\{\mathbf{U}_{0}\right\}=\lambda_{1} \mathbf{K}_{0}\left\{\mathbf{U}_{0}\right\}+\lambda_{0} \mathbf{K}_{1}\left\{\mathbf{U}_{0}\right\}+\lambda_{0} \mathbf{K}_{0}\left\{\mathbf{U}_{1}\right\} \text { in } \bar{\Omega}
$$

for the zeroth and first-order approximations, respectively. The inner products of (5.28) and (5.29) with $\mathbf{U}_{0}$ are expressed by

$$
\begin{aligned}
& \left\langle\mathbf{P}_{0}\left\{\mathbf{U}_{0}\right\}: \mathbf{U}_{0}\right\rangle=\left\langle\lambda_{0} \mathbf{K}_{0}\left\{\mathbf{U}_{0}\right\}: \mathbf{U}_{0}\right\rangle \text { in } \bar{\Omega}, \\
& \left\langle\mathbf{P}_{0}\left\{\mathbf{U}_{1}\right\}+\mathbf{P}_{1}\left\{\mathbf{U}_{0}\right\}: \mathbf{U}_{0}\right\rangle=\left\langle\lambda_{1} \mathbf{K}_{0}\left\{\mathbf{U}_{0}\right\}+\lambda_{0} \mathbf{K}_{1}\left\{\mathbf{U}_{0}\right\}+\lambda_{0} \mathbf{K}_{0}\left\{\mathbf{U}_{1}\right\}: \mathbf{U}_{0}\right\rangle \text { in } \bar{\Omega} .
\end{aligned}
$$

Subtracting one from another and then solving for $\lambda_{0}$, one has

$$
\lambda=\lambda_{0}+\varepsilon \lambda_{1}
$$

with

$$
\lambda_{1}=\left\langle\mathbf{U}_{0}: \mathbf{P}_{1}\left\{\mathbf{U}_{0}\right\}\right\rangle-\lambda_{0}\left\langle\mathbf{U}_{0}: \mathbf{K}_{1}\left\{\mathbf{U}_{0}\right\}\right\rangle /\left\langle\mathbf{U}_{0}: \mathbf{K}\left\{\mathbf{U}_{0}\right\}\right\rangle .
$$

In this equation, the solution of the zeroth order is taken to be known and then the frequency shift may be readily computed (cf. [26]-28] for a piezoelectric body).

Properties of eigenvalues and eigenfunctions. With the resulting selfadjointness of the operators $\mathbf{P}\{\mathbf{U}\}$ and $\mathbf{K}\{\mathbf{U}\}$, the eigenvalues $\lambda$ are shown to be real and positive and the orthogonality condition of the eigenvectors is obtained. First of all, one writes the inner products of (5.5) and (5.12) by $\overline{\mathbf{U}}$ and $\mathbf{U}$, respectively, as

$$
\begin{aligned}
& \langle\mathbf{P}\{\mathbf{U}\}: \overline{\mathbf{U}}\rangle=\lambda\langle\mathbf{K}\{\mathbf{U}\}: \overline{\mathbf{U}}\rangle, \\
& \langle\mathbf{P}\{\overline{\mathbf{U}}\}: \mathbf{U}\rangle=\bar{\lambda}\langle\mathbf{K}\{\overline{\mathbf{U}}\}: \mathbf{U}\rangle .
\end{aligned}
$$

By subtracting one from another and remembering the properties (5.24) and (5.26), one has

$$
(\lambda-\bar{\lambda})\langle\mathbf{K}\{\mathbf{U}\}: \overline{\mathbf{U}}\rangle=0 .
$$


By virtue of (5.14) this equation yields $\lambda=\bar{\lambda}$, that is, the eigenvalue $\lambda$ is real. Moreover, the inner product of (5.5) with $\mathbf{U}$ is expressed by

$$
\langle\mathbf{P}\{\mathbf{U}\}: \mathbf{U}\rangle=\lambda\langle\mathbf{K}\{\mathbf{U}\}: \mathbf{U}\rangle,
$$

which results in

$$
\lambda=(\langle\mathbf{P}\{\mathbf{U}\}: \mathbf{U}\rangle /\langle\mathbf{K}\{\mathbf{U}\}: \mathbf{U}\rangle)>0 .
$$

In this equation, the numerator and denominator are nonnegative for the eigenfunction $\mathbf{U}$ as shown in (5.20). Thus, all the eigenvalues $\lambda$ of (5.5) are positive.

To obtain the orthogonality condition, one considers two distinct pairs $\left(\lambda_{m}, \mathbf{U}_{m}\right)$ and $\left(\lambda_{n}, \mathbf{U}_{n}\right)$ for the solutions of (5.5), namely,

$$
\mathbf{P}\left\{\mathbf{U}_{m}\right\}=\lambda_{m} \mathbf{K}\left\{\mathbf{U}_{m}\right\}, \quad \mathbf{P}\left\{\mathbf{U}_{n}\right\}=\lambda_{n} \mathbf{K}\left\{\mathbf{U}_{\mathrm{n}}\right\} .
$$

The inner products of (5.37) respectively with $\mathbf{U}_{n}$ and $\mathbf{U}_{m}$ yield

$$
\left\langle\mathbf{P}\left\{\mathbf{U}_{m}\right\}: \mathbf{U}_{n}\right\rangle=\lambda_{m}\left\langle\mathbf{K}\left\{\mathbf{U}_{m}\right\}: \mathbf{U}_{n}\right\rangle,\left\langle\mathbf{P}\left\{\mathbf{U}_{n}\right\}: \mathbf{U}_{m}\right\rangle=\lambda_{n}\left\langle\mathbf{K}\left\{\mathbf{U}_{n}\right\}: \mathbf{U}_{m}\right\rangle .
$$

Here, by subtracting one from another, one obtains

$$
\left\langle\mathbf{P}\left\{\mathbf{U}_{m}\right\}: \mathbf{U}_{n}\right\rangle-\left\langle\mathbf{P}\left\{\mathbf{U}_{n}\right\} \mathbf{U}_{m}\right\rangle=\lambda_{m}\left\langle\mathbf{K}\left\{\mathbf{U}_{m}\right\}: \mathbf{U}_{n}\right\rangle-\lambda_{n}\left\langle\mathbf{K}\left\{\mathbf{U}_{n}\right\} \mathbf{U}_{m}\right\rangle .
$$

By virtue of the selfadjointness properties (5.24) and (5.26), this equation is reduced to

$$
\left(\lambda_{m}-\lambda_{n}\right)\left\langle\mathbf{K}\left\{\mathbf{U}_{m}\right\}: \mathbf{U}_{n}\right\rangle=0
$$

and then, to

$$
\left\langle\mathbf{K}\left\{\mathbf{U}_{m}\right\}: \mathbf{U}_{n}\right\rangle=0
$$

since $\lambda_{m} \neq \lambda_{n}$. Equation (5.41) is the orthogonality condition for the eigenvectors associated with distinct eigenvalues. The orthogonality condition is readily expressed by

$$
\left\langle\mathbf{K}\left\{\mathbf{U}_{m}\right\}: \mathbf{U}_{n}\right\rangle=\int_{\Omega} \rho\left(u_{i}^{(m)} u_{i}^{(n)}+J_{i j} \phi_{i}^{(m)} \phi_{j}^{(n)}\right) d V=0
$$

or, equivalently,

$$
\begin{aligned}
& \left\langle\mathbf{P}\left\{\mathbf{U}_{m}\right\}: \mathbf{U}_{n}\right\rangle=\int_{\Omega}\left\{\left[\mathrm{A}_{i j k l}\left(u_{l, k i}+\varepsilon_{l k s} \phi_{s, i}\right)+C_{i j k l} \phi_{j, l i}\right]^{(m)} u_{j}^{(n)}\right. \\
& +\left[\mathrm{C}_{i j k l}\left(u_{l, k i}+\varepsilon_{l k s} \phi_{s, i}\right)+B_{i j k l} \phi_{k, l i}\right]^{(m)} \phi_{j}^{(n)} \\
& \left.+\varepsilon_{j k l}\left[\mathrm{~A}_{k l r s}\left(u_{s, r}+\varepsilon_{s r i} \phi_{i}\right)+C_{k l r s} \phi_{r, s}\right]^{(m)} \phi_{j}^{(n)}\right\} d V=0
\end{aligned}
$$

in view of (5.7), (5.9) and (5.39). Similar results were presented for the free vibrations of a piezoelectromagnetic body [29].

Rayleigh's micropolar quotient. As an extension of the nonpolar case, a functional $R\{\mathbf{U}\}$ is defined in the form

$$
R\{\mathbf{U}\}=N\{\mathbf{U}\} / D\{\mathbf{U}\}
$$

in terms of $N\{\mathbf{U}\}$ and $D\{\mathbf{U}\}$ related to the potential and kinetic energies of the micropolar elastic region $\Omega+\partial \Omega$, namely,

$$
N\{\mathbf{U}\}=\langle\mathbf{P}\{\mathbf{U}\}: \mathbf{U}\rangle, D\{\mathbf{U}\}=\langle\mathbf{K}\{\mathbf{U}\}: \mathbf{U}\rangle .
$$

The stationary condition of the functional is expressed by

$$
\delta R\{\mathbf{U}\}=[D\{\mathbf{U}\} \delta N\{\mathbf{U}\}-N\{\mathbf{U}\} \delta D\{\mathbf{U}\}] / D^{2}\{\mathbf{U}\}=0,
$$


which is reduced to

$$
\chi=\delta N\{\mathbf{U}\}-R\{\mathbf{U}\} \delta D\{\mathbf{U}\}=0 .
$$

This is explicitly written as

$$
\chi=\langle\mathbf{P}\{\mathbf{U}\}: \delta \mathbf{U}\rangle+\langle\delta \mathbf{P}\{\mathbf{U}\}: \mathbf{U}\rangle-R\{\mathbf{U}\}[\langle\mathbf{K}\{\mathbf{U}\}: \delta \mathbf{U}\rangle+\langle\delta \mathbf{K}\{\mathbf{U}\}: \mathbf{U}\rangle]
$$

and then expressed by

$$
\chi=2\langle[\mathbf{P}\{\mathbf{U}\}-R\{\mathbf{U}\} \mathbf{K}\{\mathbf{U}\}]: \delta \mathbf{U}\rangle=0,
$$

because of the equality of the form

$$
\langle\delta \mathbf{P}\{\mathbf{U}\}: \mathbf{U}\rangle=\langle\mathbf{P}\{\delta \mathbf{U}\}: \mathbf{U}\rangle=\langle\mathbf{P}\{\mathbf{U}\} \delta \mathbf{U}: \mathbf{U}\rangle=\langle\mathbf{P}\{\mathbf{U}\}: \delta \mathbf{U}\rangle
$$

for the operator $\mathbf{P}\{\mathbf{U}\}$ or $\mathbf{K}\{\mathbf{U}\}$ and the selfadjointness conditions (5.24) and (5.26). From the stationary condition (5.43), it follows that

$$
\mathbf{P}\{\mathbf{U}\}-R\{\mathbf{U}\} \mathbf{K}\{\mathbf{U}\}=0
$$

by use of the fundamental lemma of the calculus of variations. In view of (5.50) and (5.5), one concludes that the stationary value of the functional $R\{\mathbf{U}\}$ gives the eigenvalue $\lambda$ provided that the divergence equations (5.1) and (5.2) are satisfied for the eigenfunctions U subjected to the boundary conditions (5.3) and (5.4) as the constraint conditions. Rayleigh's micropolar quotient is given by (5.43) together with (5.3), (5.4), (5.16) and (5.19) by

$$
\begin{aligned}
& N\{\mathbf{U}\}=\int_{\Omega}\left\{\left[A_{i j k l}\left(u_{l, k}+\varepsilon_{l k s} \phi_{s}\right)\right] u_{j, i}+\left[C_{i j k l}\left(u_{k, l}+\varepsilon_{k l s} \phi_{k, l}\right)+B_{i j k l} \phi_{k, l}\right] \phi_{j, i}\right. \\
& \left.-\varepsilon_{j k l}\left[A_{k l r s}\left(u_{s, r}+\varepsilon_{s r i} \phi_{i}\right)+C_{k l r s} \phi_{s, r}\right] \phi_{j}\right\} d V
\end{aligned}
$$

and

$$
D\{\mathbf{U}\}=\int_{\Omega} \rho\left(u_{i} u_{i}+J_{i j} \phi_{i} \phi_{j}\right) d V
$$

in terms of the deformation components and the material elasticities. The constraint conditions are undesirable as already pointed out in the previous section, and they can be removed by introducing $N_{g}$ and $D_{g}$ of the form

$$
\begin{aligned}
& N_{g}\left\{u_{i}, e_{i j}, t_{i j} ; \phi_{i}, \varepsilon_{i j}, m_{i j}\right\} \\
& =\int_{\Omega}\left\{-\left[e_{i j}-\left(u_{j, i}+\varepsilon_{j k l} \phi_{k}\right)\right] t_{i j}-\left(\varepsilon_{i j}-\phi_{i, j}\right) m_{i j}+u\left(e_{i j}, \varepsilon_{i j}\right)\right\} d V \\
& \quad-\int_{\partial \Omega_{u}} u_{i} n_{j} t_{i j} d S-\int_{\partial \Omega_{\phi}} \phi_{i} n_{j} m_{i j} d S, \\
& D_{g}\left\{u_{i}, \phi_{i}\right\}=\frac{1}{2} \int_{\Omega} \rho\left(u_{i} u_{i}+J_{i j} \phi_{i} \phi_{j}\right) d V
\end{aligned}
$$

in lieu of $N$ and $D$ in Rayleigh's quotient in (5.43). Taking the variations, integrating by parts and applying the divergence theorem to the regular region $\Omega+\partial \Omega$, one obtains

$$
\delta D_{g}=\int_{\Omega} \rho\left(u_{i} \delta u_{i}+J_{i j} \phi_{i} \phi_{j}\right) d V
$$


and

$$
\begin{aligned}
& \delta N_{g}=\int_{\Omega}\left\{-t_{i j, i} \delta u_{j}-\left(m_{i j, i}+\varepsilon_{j k l} t_{k l}\right) \delta \phi_{j}-\left[e_{i j}-\left(u_{j, i}+\varepsilon_{j k l} \phi_{k}\right)\right] \delta t_{i j}\right. \\
& \left.-\left(\varepsilon_{i j}-\phi_{i, j}\right) \delta m_{i j}-\left(t_{i j}-\frac{\partial u}{\partial e_{i j}}\right) \delta e_{i j}-\left(m_{i j}-\frac{\partial u}{\partial \varepsilon_{i j}}\right) \delta \varepsilon_{i j}\right\} d V \\
& -\int_{\partial \Omega_{u}} n_{i} u_{j} \delta t_{i j} d S+\int_{\partial \Omega_{t}} n_{i} t_{i j} \delta u_{j} d S-\int_{\partial \Omega_{\phi}} n_{i} \phi_{j} \delta m_{i j} d S+\int_{\partial \Omega_{m}} n_{i} m_{i j} \delta \phi_{j} d S .
\end{aligned}
$$

Inserting these equations into (5.46), one has the gradient equations (2.3) and (2.4), the constitutive relations (2.5) and (2.6), the boundary conditions (5.6) and the divergence equations of the form

$$
t_{i j, i}+\rho \frac{N_{g}}{D_{g}} u_{j}=0, \quad m_{i j, i}+\varepsilon_{j k l} t_{k l}+\rho J_{i j} \frac{N_{g}}{D_{g}} \phi_{i}=0 .
$$

In view of these equations together with (4.5) and (4.6), one concludes that the stationary value of $R_{g}$ gives the eigenvalue $\lambda$. The functional $R_{g}$ has no constraint conditions, and thus, all the field variables can be treated as independent variables.

In the case of a frequency shift, inserting the expansions (5.27) into the functional (5.43), one writes Rayleigh's quotient of the form

$$
\left.R\{\mathbf{U}\}=\left[\left\langle\left(\mathbf{P}_{0}\{\mathbf{U}\}+\varepsilon \mathbf{P}_{1}\{\mathbf{U}\}+\ldots\right): \mathbf{U}\right\rangle\right] /\left[\left\langle\mathbf{K}_{0}\{\mathbf{U}\}+\varepsilon \mathbf{K}_{1}\{\mathbf{U}\}+\ldots\right): \mathbf{U}\right\rangle\right] .
$$

By use of the unperturbed mode $\mathbf{U}_{0}$ as an approximating function in this equation, one reads

$$
\lambda \approx\left[\left\langle\left(\mathbf{P}_{0}\left\{\mathbf{U}_{0}\right\}+\varepsilon \mathbf{P}_{1}\left\{\mathbf{U}_{0}\right\}\right): \mathbf{U}_{0}\right\rangle\right] /\left[\left\langle\left(\mathbf{K}_{0}\left\{\mathbf{U}_{0}\right\}+\varepsilon \mathbf{K}_{1}\left\{\mathbf{U}_{0}\right\}\right): \mathbf{U}_{0}\right\rangle\right],
$$

which is expressed by

$$
\lambda \approx\left[\left\langle\mathbf{P}_{0}\left\{\mathbf{U}_{0}\right\}: \mathbf{U}_{0}\right\rangle+\varepsilon\left\langle\mathbf{P}_{1}\left\{\mathbf{U}_{0}\right\}: \mathbf{U}_{0}\right\rangle\right] /\left[\left\langle\left(\mathbf{K}_{0}\left\{\mathbf{U}_{0}\right\}+\varepsilon \mathbf{K}_{1}\left\{\mathbf{U}_{0}\right\}\right): \mathbf{U}_{0}\right\rangle\right] .
$$

This equation is approximated by

$$
\lambda \approx \lambda_{0}\left[1+\varepsilon \mathbf{P}_{1}\left\{\mathbf{U}_{0}\right\} / \mathbf{P}_{0}\left\{\mathbf{U}_{0}\right\}\right]\left[1-\varepsilon \mathbf{K}_{1}\left\{\mathbf{U}_{0}\right\} / \mathbf{K}_{0}\left\{\mathbf{U}_{0}\right\}\right] ; \lambda_{0}=\mathbf{P}_{0}\left\{\mathbf{U}_{0}\right\} / \mathbf{K}_{0}\left\{\mathbf{U}_{0}\right\} .
$$

From this equation, the eigenvalue $\lambda$ may be expressed by

$$
\left.\lambda \approx \lambda_{0}+\varepsilon\left[\left\langle\mathbf{P}_{1}\left\{\mathbf{U}_{0}\right\}: \mathbf{U}_{0}\right\rangle\right]-\lambda_{0}\left\langle\mathbf{K}_{1}\left\{\mathbf{U}_{0}\right\}: \mathbf{U}_{0}\right\rangle\right] /\left\langle\mathbf{K}_{0}\left\{\mathbf{U}_{0}\right\}: \mathbf{U}_{0}\right\rangle,
$$

where (5.24) and (5.26) are used. This eigenvalue is equal to that in (5.31) for the first-order approximation.

6. Free vibrations of a micropolar elastic plate. In the Euclidean space $\Xi$, consider a micropolar thin elastic plate of uniform thickness $2 h$. The plate is referred to the Cartesian rectangular coordinates $x_{i}$ with the faces of area $A$, at $x_{3}= \pm h$ and with $x_{\alpha}$ the coordinates on the middle plane $\left(x_{3}=0\right)$ which intersects the right cylindrical or primary boundary surface of the plate in a Jordan curve $C$. The Jordan curve consists of the complementary curves $C_{u}, C_{t}, C_{\phi}$ and $C_{m}$ (i.e., $C=C_{u} \cup C_{t}=C_{\phi} \cup C_{m}$ and $\left.C_{u} \cap C_{t}=C_{\phi} \cap C_{m}=\phi\right)$ and the unit outward vector normal to $C$ is denoted by $\nu_{\alpha}$. 
The equations governing the free high-frequency vibrations of the plate are derived in differential form on the basis of the power series expansions by

$$
\left\{u_{i}\left(x_{j}\right), \phi_{i}\left(x_{j}\right)\right\}=\sum_{n=0}^{N=\infty}\left\{u_{i}^{(n)}\left(x_{\alpha}\right), \phi_{i}\left(x_{\alpha}\right)\right\} z^{n}, \quad z \equiv x_{3}
$$

for the deformation components and following Mindlin's method of reduction in plates [30. Accordingly, inserting the expansions (6.1) into the variational principle (4.14), and then performing the integrations across the thickness interval $Z=[-h, h]$, one obtains the 2-D governing equations of the micropolar plates, as the Euler-Lagrange equations, by

Field equations.

$$
\begin{gathered}
-T_{\alpha j, \alpha}^{(n)}+n T_{3 j}^{(n-1)}-T_{j}^{(n)}=\rho \lambda_{p} \sum_{m=0}^{N} I_{m n} u_{j}^{(m)} \text { on } A \\
-M_{\alpha j, \alpha}^{(n)}+n M_{3 j}^{(n-1)}-M_{j}^{(n)}-\varepsilon_{j k l} T_{k l}^{(n)}=\rho \lambda_{p} J_{i j} \sum_{m=0}^{N} I_{m n} \phi_{i}^{(m)} \text { on } A
\end{gathered}
$$

where

$$
\begin{aligned}
& \left(T_{i j}^{(n)}, M_{i j}^{(n)}\right)=\int_{Z}\left(t_{i j}, m_{i j}\right) z^{n} d z, \quad Z=[-h, h], \\
& \left(T_{i}^{(n)}, M_{i}^{(n)}\right)=\left[\left(t_{3 i}, m_{3 i}\right) z^{n}\right]_{-h}^{h}=0, \\
& I_{m n}=\frac{2 h^{(m+n+1)}}{m+n+1}, m+n=2 p \text { (even). }
\end{aligned}
$$

Gradient equations.

$$
\begin{aligned}
& -e_{i j}^{(n)}+u_{j, i}^{(n)}+(n+1) u_{j}^{(n+1)} \delta_{3 i}+\varepsilon_{j i k} \phi_{k}=0 \text { on } A, \\
& -\varepsilon_{i j}^{(n)}+\phi_{i, j}^{(n)}+(n+1) \phi_{j}^{(n+1)} \delta_{3 i}=0 \text { on } A .
\end{aligned}
$$

Constitutive relations.

$$
-T_{i j}^{(n)}+\frac{\partial H}{\partial e_{i j}^{(n)}}=0, \quad-M_{i j}^{(n)}+\frac{\partial H}{\partial \varepsilon_{i j}^{(n)}}=0 .
$$

Here, all the quantities are functions of the aerial coordinates $x_{\alpha}$ of the plate, and the elastic energy $H$ per unit area of the middle plane $A$ is given by

$$
H=\int_{Z} u d z
$$

A quadratic form of $H$ is recorded as

$$
H=\frac{1}{2} \sum_{m=0}^{N} \sum_{n=0}^{N} I_{m n}\left(A_{i j k l} e_{i j}^{(m)} e_{k l}^{(n)}+B_{i j k l} \varepsilon_{i j}^{(m)} \varepsilon_{k l}^{(n)}+2 C_{i j k l} e_{i j}^{(m)} \varepsilon_{k l}^{(n)}\right) .
$$

This leads to the linear constitutive relations of the form

$$
\begin{aligned}
& T_{i j}^{(n)}=\sum_{m=0}^{N}\left(A_{i j k l} \varepsilon_{k l}^{(m)}+C_{i j k l} \varepsilon_{k l}^{(m)}\right) I_{m n} \text { on } A, \\
& M_{i j}^{(n)}=\sum_{m=0}^{N}\left(C_{i j k l} e_{k l}^{(m)}+B_{i j k l} \varepsilon_{k l}^{(m)}\right) I_{m n} \text { on } A .
\end{aligned}
$$


Boundary conditions.

$$
\begin{aligned}
& u_{i}^{(n)}=0 \text { on } C_{u}, \quad \nu_{\alpha} T_{\alpha j}^{(n)}=0 \text { on } C_{t}, \\
& \phi_{i}^{(n)}=0 \text { on } C_{\phi}, \quad \nu_{\alpha} M_{\alpha j}^{(n)}=0 \text { on } C_{m} .
\end{aligned}
$$

In the foregoing equations, the superscript $(n)$ is used to indicate the $n$ th-order components of a quantity of the functions $x_{\alpha}$.

The 2-D equations (6.2)-(6.10) govern all the types of high frequency vibrations of the micropolar plate. They form a deterministic system of $42 N$ equations with $42 N$ dependent variables $\left(u_{i}^{(n)}, e_{i j}^{(n)}, T_{i j}^{(n)}, \phi_{i}^{(n)}, \varepsilon_{i j}^{(n)}, M_{i j}^{(n)}\right)$ of functions of the aerial coordinates $x_{\alpha}$. The boundary conditions (6.10) were shown to be sufficient for a unique solution in 31, where the vibration of a micropolar shell is treated.

The governing equations define an eigenvalue problem for the values $\lambda_{p}=\omega_{p}^{2}$, which correspond to a nontrivial solution of the $n$ th-order dependent variables. Alternatively, the eigenvalue problem may be defined in an abstract form by

$$
\mathbf{P}_{p}\left\{\mathbf{U}_{p}\right\}=\lambda_{p} \mathbf{K}_{p}\left\{\mathbf{U}_{p}\right\} .
$$

Here, the field vector given by

$$
\mathbf{U}_{p}=\left\{u_{i}^{(n)}, e_{i j}^{(n)}, T_{i j}^{(n)}, \phi_{i}^{(n)}, \varepsilon_{i j}^{(n)}, M_{i j}^{(n)}\right\},
$$

the operators given by

$$
\begin{gathered}
\mathbf{P}_{p}\left\{\mathbf{U}_{p}\right\}=\left\{-T_{\alpha j, \alpha}^{(n)}+n T_{3 j}^{(n-1)},-e_{i j}^{(n)}+u_{j, i}^{(n)}+(n+1) u_{j}^{(n+1)} \delta_{3 i}+\varepsilon_{j i k} \phi_{k}^{(n)},-T_{i j}^{(n)}\right. \\
+\frac{\partial H}{\partial e_{i j}^{(n)}} \\
\left.-M_{\alpha j, \alpha}^{(n)}+n M_{3 j}^{(n-1)}-\varepsilon_{j k l} T_{k l}^{(n)},-\varepsilon_{i j}^{(n)}+\phi_{i, j}^{(n)}+(n+1) \phi_{j}^{(n+1)} \delta_{3 i},-M_{i j}^{(n)}+\frac{\partial H}{\partial \varepsilon_{i j}^{(n)}}\right\} \\
\mathbf{K}_{p}\left\{\mathbf{U}_{p}\right\}=\left\{\rho \sum_{m=0}^{N} I_{m n} u_{j}^{(m)}, 0,0 ; \rho J_{i j} \sum_{m=0}^{N} I_{m n} \phi_{i}^{(m)}, 0,0\right\}
\end{gathered}
$$

and the function space given by

$$
\Gamma_{p}=\mathbf{U}_{p} \cup \overline{\mathbf{U}}_{p}
$$

are introduced for the micropolar plate. Also, a function space $\mathbf{V}_{p} \in \Gamma$ given by

$$
\mathbf{V}_{p}=\left\{v_{i}^{(n)}, E_{i j}^{(n)}, \sigma_{i j}^{(n)} ; \varphi_{i}^{(n)}, S_{i j}^{(n)}, N_{i j}^{(n)}\right\} .
$$

A quantity similar to that in (6.8) given by

$$
I=\frac{1}{2} \sum_{m=0}^{N} \sum_{n=0}^{N} I_{m n}\left(A_{i j k l} E_{i j}^{(m)} E_{i j}^{(n)}+B_{i j k l} S_{i j}^{(m)} S_{k l}^{(n)}+2 C_{i j k l} E_{i j}^{(m)} S_{k l}^{(n)}\right),
$$

which are functions of the aerial coordinates $x_{\alpha}$, are defined. 
The inner product of the operator $\mathbf{P}_{p}\left\{\mathbf{U}_{p}\right\}$ and the function space $V_{p}$ is given by

$$
\begin{aligned}
& \left\langle\mathbf{P}_{p}\left\{\mathbf{U}_{p}\right\}: \mathbf{V}_{p}\right\rangle=\sum_{n=0}^{N} \int_{A}\left\{\left(-T_{\alpha j, \alpha}^{(n)}+n T_{3 j}^{(n-1)}\right) v_{j}^{(n)}\right. \\
& +\left[-e_{i j}^{(n)}+u_{j, i}^{(n)}+(n+1) u_{j}^{(n+1)} \delta_{3 i}+\varepsilon_{j i k} \phi_{k}^{(n)}\right] \sigma_{i j}^{(n)} \\
& +\left(-T_{i j}^{(n)}+\frac{\partial H}{\partial e_{i j}^{(n)}}\right) E_{i j}^{(n)}+\left(-M_{\alpha j, \alpha}^{(n)}+n M_{3 j}^{(n-1)}-\varepsilon_{j k l} \phi_{k}^{(n)}\right) \varphi_{j}^{(n)} \\
& \left.+\left[-\varepsilon_{i j}^{(n)}+\phi_{i, j}^{(n)}+(n+1) \phi_{j}^{(n+1)}\right] N_{i j}^{(n)}+\left(-M_{i j}^{(n)}+\frac{\partial H}{\partial \varepsilon_{i j}^{(n)}}\right) S_{i j}^{(n)}\right\} d A .
\end{aligned}
$$

This equation is evaluated through integration by parts and then applying the divergence theorem to the plate region in a similar manner to (5.21) with the result

$$
\begin{aligned}
& \left\langle\mathbf{P}_{p}\left\{\mathbf{U}_{p}\right\}: \mathbf{V}_{p}\right\rangle=\sum_{n=0}^{N} \int_{A}\left\{\left(-\sigma_{\alpha j, \alpha}^{(n)}+n \sigma_{3 j}^{(n-1)}\right) u_{j}^{(n)}\right. \\
& +\left[-E_{i j}^{(n)}+v_{j, i}^{(n)}+(n+1) v_{j}^{(n+1)} \delta_{3 i}+\varepsilon_{j i k} \varphi_{k}^{(n)}\right] T_{i j}^{(n)} \\
& +\left(-\sigma_{i j}^{(n)}+\frac{\partial \mathrm{I}}{\partial E_{i j}^{(n)}}\right) e_{i j}^{(n)}+\left(-N_{\alpha j, \alpha}^{(n)}+n N_{3 j}^{(n-1)}-\varepsilon_{j k l} \varphi_{k}^{(n)}\right) \phi_{j}^{(n)} \\
& \left.+\left[-S_{i j}^{(n)}+\varphi_{i, j}^{(n)}+(n+1) \varphi_{j}^{(n+1)}\right] M_{i j}^{(n)}+\left(-N_{i j}^{(n)}+\frac{\partial I}{\partial S_{i j}^{(n)}}\right) \varepsilon_{i j}^{(n)}\right\} d A \\
& \quad=\left\langle\mathbf{P}_{p}\left\{\mathbf{V}_{p}\right\}: \mathbf{U}_{p}\right\rangle
\end{aligned}
$$

and the boundary conditions (6.10) are considered. Likewise, the inner product of the operator $\mathbf{K}_{p}\left\{\mathbf{U}_{p}\right\}$ and the function space $\mathbf{V}_{p}$ is given by

$$
\left\langle\mathbf{K}\left\{\mathbf{U}_{p}\right\}: \mathbf{V}_{p}\right\rangle=\rho \sum_{m=0}^{N} \sum_{n=0}^{N} \int_{A} I_{m n}\left(u_{i}^{(m)} v_{i}^{(n)}+J_{i j} \phi_{i}^{(m)} \varphi_{j}^{(n)}\right) d A=\left\langle\mathbf{K}\left\{\mathbf{V}_{p}\right\}: \mathbf{U}_{p}\right\rangle .
$$

The conditions (6.19) and (6.20) show the selfadjointness of the operators $\mathbf{P}_{p}\left\{\mathbf{U}_{p}\right\}$ and $\mathbf{K}_{p}\left\{\mathbf{U}_{p}\right\}$. With the selfadjointness properties of the operators, the reality and positivity of the plate eigenvalues can be proved as in the 3-D case in (5.34) and (5.36).

The governing equations (6.11) are expressed by

$$
\mathbf{P}_{p}\left\{\mathbf{U}_{p}^{(r)}\right\}=\lambda_{p}^{(r)} \mathbf{K}_{p}\left\{\mathbf{U}_{p}^{(r)}\right\}, \mathbf{P}_{p}\left\{\mathbf{U}_{p}^{(s)}\right\}=\lambda_{p}^{(s)} \mathbf{K}_{p}\left\{\mathbf{U}_{p}^{(s)}\right\}
$$

at two distinct eigenvalues $\lambda_{p}^{(r)}$ and $\lambda_{p}^{(s)}$, which respectively correspond to eigenfunctions $\mathbf{U}_{p}^{(r)}$ and $\mathbf{U}_{p}^{(s)}$. Taking the inner products of these equations by $\mathbf{U}_{p}^{(s)}$ and $\mathbf{U}_{p}^{(r)}$ and then subtracting one from another as in (5.40) for the 3-D case and considering the selfadjointness property of the operators, one writes

$$
\left(\lambda_{p}^{(s)}-\lambda_{p}^{(r)}\right)\left\langle\mathbf{K}_{p}\left\{\mathbf{U}_{p}^{(r)}\right\}: \mathbf{U}_{p}^{(s)}\right\rangle=0
$$

It follows from this equation that

$$
\left\langle\mathbf{K}_{p}\left\{\mathbf{U}_{p}^{(r)}\right\}: U_{p}^{(s)}\right\rangle=0
$$

since $\lambda_{p}^{(r)} \neq \lambda_{p}^{(s)}$. This equation is the orthogonality condition for the eigenvectors of the micropolar plate. The condition is explicitly given by

$$
\left\langle\mathbf{K}_{p}\left\{\mathbf{U}_{p}^{(r)}\right\}: \mathbf{U}_{p}^{(s)}\right\rangle=\rho \sum_{n=0}^{N} \sum_{m=0}^{N} \int_{A} I_{m n}\left(u_{i}^{(m) r} u_{i}^{(n) s}+J_{i j} \phi_{i}^{(m) r} \phi_{j}^{(n) s}\right) d A=0
$$


and also, by

$$
\begin{aligned}
\left\langle\mathbf{P}_{p}\right. & \left.\left\{\mathbf{U}_{p}^{(r)}\right\}: \mathbf{U}_{p}^{(s)}\right\rangle \\
= & \sum_{n=0}^{N} \sum_{m=0}^{N} \int_{A}\left\{\left(-T_{\alpha j, \alpha}^{(n)}+n T_{3 j}^{(n-1)}\right)^{r} u_{j}^{(m) s}+\left(-T_{i j}^{(n)}+\frac{\partial H}{\partial e_{i j}^{(n)}}\right)^{r} e_{i j}^{(m) s}\right. \\
& +\left[-e_{i j}^{(n)}+u_{j, i}^{(n)}+(n+1) u_{j}^{(n+1)} \delta_{3 i}+\varepsilon_{j i k} \phi_{k}^{(n)}\right]^{\mathrm{r}} T_{i j}^{(m) s}+\left(-M_{i j}^{(n)}+\frac{\partial H}{\partial \varepsilon_{i j}^{(n)}}\right)^{r} \varepsilon_{i j}^{(m) s} \\
& +\left(-M_{\alpha j, \alpha}^{(n)}+n M_{3 j}^{(n-1)}-\varepsilon_{j k l} \phi_{k}^{(n)}\right)^{r} \phi_{j}^{(m) s} \\
& \left.+\left[-\varepsilon_{i j}^{(n)}+\phi_{i, j}^{(n)}+(n+1) \phi_{j}^{(n+1)}\right]^{r} M_{i j}^{(m) s}\right\} d A .
\end{aligned}
$$

Here, $H$ is obtained by substituting (6.5) into (6.7) with (2.7) and then integrating across the plate as

$$
H=\frac{1}{2} \sum_{m=0}^{N} \sum_{n=0}^{N} I_{m n}\left(A_{i j k l} e_{i j}^{(m)} e_{k l}^{(n)}+B_{i j k l} \varepsilon_{i j}^{(m)} \epsilon_{k l}^{(n)}+2 C_{i j k l} e_{i j}^{(m)} \varepsilon_{k l}^{(n)}\right) .
$$

Furthermore, the condition (6.24) can be expressed by

$\sum_{n=0}^{N} \sum_{m=0}^{N} \int_{A}\left[\left(-T_{\alpha j, \alpha}^{(n)}+n T_{3 j}^{(n-1)}\right)^{r} u_{j}^{(m) s}+\left(-M_{\alpha j, \alpha}^{(n)}+n M_{3 j}^{(n-1)}-\varepsilon_{j k l} T_{k l}^{(n)}\right)^{r} \phi_{j}^{(m) s}\right] d A=0$,

where (6.2) and (6.3) are considered.

Rayleigh's quotient for the micropolar plate. With the help of (6.1), Rayleigh's quotient is readily stated by

$$
R\left\{\mathbf{U}_{p}\right\}=\left\langle\mathbf{P}_{p}\left\{\mathbf{U}_{p}\right\}: \mathbf{U}_{p}\right\rangle /\left\langle\mathbf{K}_{p}\left\{\mathbf{U}_{p}\right\}: \mathbf{U}_{p}\right\rangle,
$$

which is given explicitly by

$$
\begin{aligned}
& R\left\{\mathbf{U}_{p}\right\}=\sum_{n=0}^{N} \sum_{m=0}^{N} \int_{A}\left\{\left(T_{\alpha j}^{(n)} u_{j, \alpha}^{(n)}+n T_{3 j}^{(n-1)}\right) u_{j}^{(m)}\right. \\
& +M_{\alpha j}^{(n)} \phi_{j, \alpha}^{(m)}+\left(n M_{3 j}^{(n-1)}-\varepsilon_{j k l} T_{k l}^{(n)}\right) \phi_{j}^{(m)} \\
& +\left[-e_{i j}^{(n)}+u_{j, i}^{(n)}+(n+1) u_{j}^{(n+1)} \delta_{3 i}+\varepsilon_{j i k} \phi_{k}^{(n)}\right] T_{i j}^{(m)} \\
& +\left[-\varepsilon_{i j}^{(n)}+\phi_{i, j}^{(n)}+(n+1) \phi_{j}^{(n+1)} \delta_{3 i}\right] M_{i j}^{(m)}{ }^{(n)} \\
& \left.+\left(-T_{i j}^{(n)}+\frac{\partial H}{\partial e_{i j}^{(n)}}\right) e_{i j}^{(m)}+\left(-M_{i j}^{(n)}+\frac{\partial H}{\partial \varepsilon_{i j}^{(n)}}\right) \varepsilon_{i j}^{(m)}\right\} d A \\
& \\
& \quad / \frac{1}{2} \rho \sum_{n=0}^{N} \sum_{m=0}^{N} I_{m n} \int_{A}\left(u_{i}^{(n)} u_{j}^{(m)}+J_{i j} \phi_{i}^{(n)} \phi_{j}^{(m)}\right) d A,
\end{aligned}
$$

where pertinent integration by parts is made, and the deformation boundary conditions are considered.

7. Conclusions. This paper is addressed to the 3 -D equations governing the timeharmonic free vibrations of a polar elastic body, including the uniqueness of solutions, the variational formulations and the example of a micropolar plate. The boundary conditions sufficient to the uniqueness are enumerated by appealing to the logarithmic convexity 
argument instead of the classical energy argument that imposes the positive-definiteness assumption of material elasticities. The variational principles of the vibrations are deduced from the principle of virtual work by modifying it through an involutory transformation. A unified variational principle operating on all the field variables is shown to generate, as its Euler-Lagrange equations, all the governing equations, unlike the Hellinger-Reissner-Washizu variational principle in elasticity where the symmetry of the stress tensor always remains as a constraint condition. The vibration of a micropolar elastic body is treated, and its eigenvalue problem is defined by an abstract formulation based on the two operators related to the kinetic and potential energies of the body. The selfadjointness and nonnegativeness of the operators and the reality and positivity of eigenvalues are shown, and the orthogonality condition of eigenvectors is found. Also, Rayleigh's quotient with its well-known features is obtained for the micropolar elastic body and the frequency shift is studied by a perturbation method.

As an example, the equations governing the high-frequency vibrations of a micropolar thin elastic plate are presented. The eigenvalue problem is stated for the vibrations by an abstract formulation with the help of the 2-D operators. Certain fundamental properties of the operators and eigenvalues are examined, the orthogonality condition of eigenvectors is obtained, and Rayleigh's quotient is given for the plate.

In closing, omitting the terms involving the micropolar field, the results are readily reducible to those established in 1-D/2-D nonpolar (classical) elasticity, and, in the light of the present treatment, the free vibrations of time- and/or temperature-dependent materials subjected to the mechanical, electrical and magnetic effects can be similarly investigated, including random vibrations; some of them with numerical examples will be reported in the forthcoming works.

\section{REFERENCES}

[1] Cosserat, E. \& F. Théorie des corps deformables, Herman et Fils, Paris, 1909.

[2] C. Truesdell; Noll, W., The non-linear field theories of mechanics, Handbuch der Physik, SpringerVerlag, Berlin, 1965. MR0193816 (33:2030)

[3] Gauthier, R.D., Experimental investigations on micropolar media, in: O. Brulin, R.K.T. Hsieh (Eds.), Mechanics of micropolar media, World Scientific, Singapore,1982, pp. 395-463.

[4] Chen, Y.; Lee, J.D., Determining material constants in micromorphic theory through phonon dispersion relations, Int. J. Eng. Sci. 41(2003) 871-886.

[5] Kunin, I.A., Elastic Media with microstructure, vol. 1: One-dimensional models, vol. 2: Threedimensional models, Springer-Verlag, New York, 1982-1983. MR0664203 (84d:73008) MR0710127 (85d:73030)

[6] Nowacki, W., Theory of asymmetric elasticity, Pergamon Press, Oxford, 1986. MR0894254 (88d:73002)

[7] Eringen, A.C., Microcontinuum field theories, I. Foundations and solids, Springer-Verlag, 1999. MR 1720520 (2000i:74007)

[8] Capriz, G., Continua with microstructure, Springer-Verlag, New York, $1989 . \quad$ MR0985585 (90c:73007)

[9] Erofeyev, V.I., Wave processes in solid with microstructure, World Scientific, London, 2003.

[10] Pabst, W., Micropolar materials, Ceramics-Silikaty 49(2005), no.3, 170-180.

[11] Shahinpoor, M.; Ahmadi,G., Uniqueness in elastodynamics of Cosserat and micropolar media, Quart. Appl. Math. 31 (1973), 257-261. MR0411315 (53:15052)

[12] Altay, G.; Dökmeci, M. C., Vibrations of 1-D/2-D micropolar elastic continua, ITU and BU, TR 7, November 2001.

[13] Reissner, E., A note on variational principles in elasticity, Int. J. Solids Struct. 1 (1965) 93-95. 
[14] Dökmeci, M.C., Dynamic variational principles for discontinuous elastic fields, J. Ship Res. 23 (1979) 115-122.

[15] Felippa, C.A., Parametrized variational principles for micropolar elasticity, Int. J. Solids Struct. 29 (1992) 2709-2721. MR 1185909

[16] Steinmann, P.; Stein, E., A unifying treatise of variational principles for two types of micropolar continua, Acta Mechanica 121 (1997) 215-232. MR1455162 (98e:73004)

[17] Dyszlewicz, J., Micropolar theory of elasticity, Springer-Verlag, New York, 2004. MR2058885 (2005a:74007)

[18] Love, A.E.H., The mathematical theory of elasticity, Dover Publishers, New York, 1944. MR0010851 $(6: 79 \mathrm{e})$

[19] Altay, G.; Dökmeci, M.C., Fundamental equations of certain electromagnetic-acoustic discontinuous fields in variational form, Continuum Mech. Thermodyn.16 (2004) 53-71. MR2035527 (2004j:74042)

[20] Knops, R.J.; Payne, L.E., Uniqueness Theorems in Linear Elasticity, Springer-Verlag, New York, 1971. MR0421244 (54:9249)

[21] Altay, G.; Dökmeci, M.C., A uniqueness theorem in Biot's poroelasticity theory, Z. Angew. Math. Phys. (ZAMP) 49 (1998) 838-846. MR 1652204 (99h:73018)

[22] Altay, G.; Dökmeci, M.C., Fundamental variational equations of discontinuous thermopiezoelectric fields, Int. J. Eng. Sci. 34 (1996) 769-783.

[23] Truesdell, C.; Toupin, R.A., The classical field theories, Handbuch der Physik, Springer-Verlag, New York, 1960. MR0118005 (22:8778)

[24] Lanczos, C., The variational principles of mechanics, Univ. Toronto Press, Toronto, 1964.

[25] Dökmeci, M.C., Certain integral and differential types of variational principles in nonlinear piezoelectricity, IEEE Trans. Ultrason. Ferroelec. Freq. Cont. UFFC 35 (1988) 775-787.

[26] Yang, J.S.; Batra, R.C., Free vibrations of a piezoelectric body, J. Elasticity 34 (1994) 239-254.

[27] Tiersten, H.F., Linear piezoelectric plate vibrations, Plenum Press, New York, 1969.

[28] Yang, J.S., Variational formulations for the vibration of a piezoelectric body, Quart. Appl. Math. 53 (1995) 95-104. MR1315450 (96a:73057)

[29] Yang, J.S.; Wu, X.Y., The vibration of an elastic dielectric with piezoelectromagnetism, Quart. Appl. Math. 53 (1995), no. 4, 753-760. MR.1359509 (96f:73039)

[30] Deresiewicz, H.;Bieniek, M.P.; DiMaggio, F.L. (Eds.), The collected papers of Raymond D. Mindlin, vols. I and II, Springer-Verlag, Berlin, 1989.

[31] Altay, G.; Dökmeci, M.C., A polar theory for vibrations of thin elastic shells, Int. J. Solids Struct. 43 (2006) 2578-2601. 